\title{
OPTIMIZED SCHWARZ METHODS FOR CIRCULAR DOMAIN DECOMPOSITIONS WITH OVERLAP*
}

\author{
MARTIN J. GANDER ${ }^{\dagger}$ AND YINGXIANG XU ${ }^{\ddagger}$
}

\begin{abstract}
Optimized Schwarz methods are based on transmission conditions between subdomains which are optimized for the problem class that is being solved. Such optimizations have been performed for many different types of partial differential equations, but were almost exclusively based on the assumption of straight interfaces. We study in this paper the influence of curvature on the optimization, and we obtain four interesting new results: first, we show that the curvature does indeed enter the optimized parameters and the contraction factor estimates. Second, we develop an asymptotically accurate approximation technique, based on Turán type inequalities in our case, to solve the much harder optimization problem on the curved interface, and this approximation technique will also be applicable to currently too complex best approximation problems in the area of optimized Schwarz methods. Third, we show that one can obtain transmission conditions from a simple circular model decomposition which have also been found using microlocal analysis but that these are not the best choices for the performance of the optimized Schwarz method. Finally, we find that in the case of curved interfaces, optimized Schwarz methods are not necessarily convergent for all admissible parameters. Our optimization leads, however, to parameter choices that give the same good performance for a circular decomposition as for a straight interface decomposition. We illustrate our analysis with numerical experiments.
\end{abstract}

Key words. optimized Schwarz method, optimized transmission conditions, circular domain decomposition, interface curvature, parallel computation

AMS subject classifications. $65 \mathrm{~N} 55,65 \mathrm{~F} 10$

DOI. $10.1137 / 130946125$

1. Introduction. Domain decomposition methods are important techniques for the numerical simulation of large scale physical problems, since they can systematically reduce their complexity by decomposition and lead to efficient parallel solvers. Among the many domain decomposition methods, optimized Schwarz methods, going back to an idea for a nonoverlapping method by Lions [28], have attracted substantial attention over the past decade because their transmission conditions can be adapted to the physics of the underlying problem and thus lead to very efficient methods for hard problems; for an overview and references, see [35, 17]. Optimized Schwarz methods are a very active area of research, and they have found their way into many areas of applications: for Helmholtz problems, see [12, 6, 22], for advection diffusion evolution problems, see [34, 20, 7, 40], for wave equations, see [21, 19], for Maxwell problems, see $[41,13,38,37,8]$, and for shallow water and the primitive equations, see $[39,3]$. Optimized Schwarz methods have also led to new theoretical developments (see, for example, $[27,30,29]$ ) and many innovative preconditioners that appeared under different names; see, for example, the sweeping preconditioner in [16, 2, 23, 14], the source transfer method in [11], and also [42], which are among the best iterative

*Received by the editors November 21, 2013; accepted for publication (in revised form) June 18, 2014; published electronically August 12, 2014.

http://www.siam.org/journals/sinum/52-4/94612.html

†Section de Mathématiques, Université de Genève, CP 64, CH-1211, Genève, Switzerland (Martin.Gander@unige.ch).

${ }^{\ddagger}$ Corresponding author. School of Mathematics and Statistics, Northeast Normal University, Changchun 130024, China (yxxu@nenu.edu.cn). The research of this author was supported by NSFC-11201061, CPSF-2012M520657, and the Science and Technology Development Planning of Jilin Province 20140520058JH. 
solvers for the difficult class of Helmholtz problems. For the precise relation between those methods, see [10].

Optimized Schwarz methods are typically developed and analyzed on model domains with straight interfaces using Fourier techniques. However, the methods also work well in more general settings in many applications; see, for example, [17] for the temperature distribution in an apartment in Montreal and $[22,33]$ for the noise levels in a car compartment. A more general analysis in [31] shows that the asymptotic choice of $O\left(h^{-\frac{1}{2}}\right)$ of the Robin parameter ( $h$ being the local mesh size) will result in a contraction factor of the form $1-O\left(h^{\frac{1}{2}}\right)$ for a nonoverlapping Schwarz method. Similar results were obtained for higher order transmission conditions [32].

Since the curvature is the most important information for a smooth curve, in this paper we are interested in studying the influence of the curvature on the optimization of the transmission conditions in optimized Schwarz methods. However, it is not possible to directly study the fully general case of an arbitrarily curved interface. We thus study in detail the model problem of a circular domain decomposition and determine how the best choice of coefficients in the transmission conditions depends on the curvature explicitly. The transmission conditions we obtain can then be employed in the case of a generally curved interface by using the local curvature at each point of the interface in the best parameter formulas we obtained. They therefore have many potential applications - for example, the simulation of fluid-structure interaction problems in haemodynamics [36], where optimized Robin parameters are observed to be numerically efficient. The best approximation problem for the circular domain decomposition is however still too hard to be analyzed directly, and we present for the first time an asymptotically accurate approximation of such problems based on Turán-type inequalities. This new idea of approximation will also be important for other best approximation problems that have so far remained unsolved. It allows us to present a complete asymptotic analysis for a circular model problem which will reveal, in contrast to the straight interface case, that the optimized Schwarz algorithm is not convergent for all admissible parameters. The study of our circular model problem also allows us to obtain transmission conditions found using the completely different approach of microlocal analysis [5] and reveals that these do not lead to the best possible performance of the algorithm.

In order to obtain these results, we consider the negative definite model problem

$$
(\Delta-\eta) u=f \text { on } \Omega=\mathbb{R}^{2}, \eta>0,
$$

with a circular decomposition of $\Omega=\Omega_{1} \cup \Omega_{2}$, where for $L>0$

$$
\begin{aligned}
& \Omega_{1}=\left\{(x, y) \mid \sqrt{x^{2}+y^{2}}<R_{1}=R+L\right\}, \\
& \Omega_{2}=\left\{(x, y) \mid R_{2}=R<\sqrt{x^{2}+y^{2}}<\infty\right\} ;
\end{aligned}
$$

see Figure 1 (left).

In polar coordinates, the model problem (1.1) reads

$$
\partial_{r r} u+\frac{1}{r} \partial_{r} u+\frac{1}{r^{2}} \partial_{\theta \theta} u-\eta u=f .
$$

A general parallel Schwarz algorithm for (1.3) with decomposition (1.2) is

$$
\begin{aligned}
\partial_{r r} u_{1}^{n}+\frac{1}{r} \partial_{r} u_{1}^{n}+\frac{1}{r^{2}} \partial_{\theta \theta} u_{1}^{n}-\eta u_{1}^{n} & =f & & \text { in } \Omega_{1}, \\
\mathcal{B}_{1}\left(u_{1}^{n}\right) & =\mathcal{B}_{1}\left(u_{2}^{n-1}\right) & & \text { on } r=R_{1}, \\
\partial_{r r} u_{2}^{n}+\frac{1}{r} \partial_{r} u_{2}^{n}+\frac{1}{r^{2}} \partial_{\theta \theta} u_{2}^{n}-\eta u_{2}^{n} & =f & & \text { in } \Omega_{2}, \\
\mathcal{B}_{2}\left(u_{2}^{n}\right) & =\mathcal{B}_{2}\left(u_{1}^{n-1}\right) & & \text { on } r=R_{2} .
\end{aligned}
$$



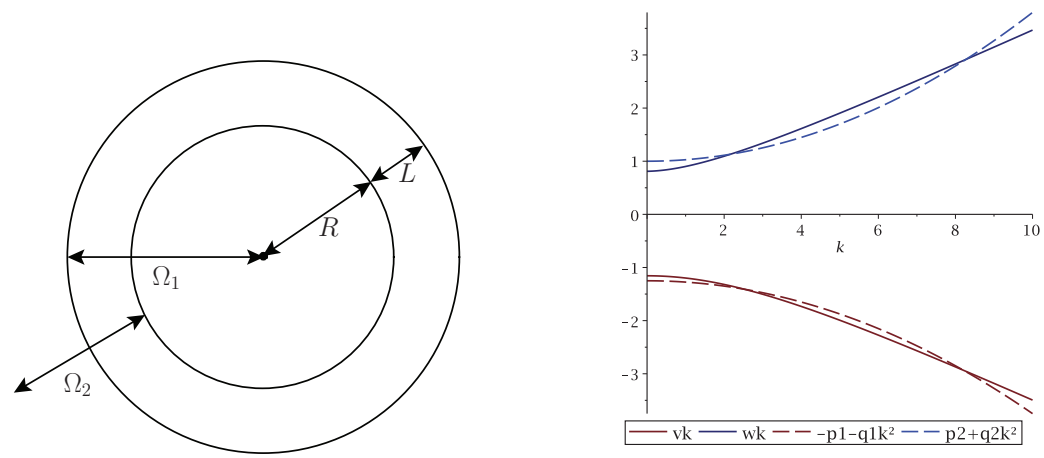

FIG. 1. Circular domain decomposition on the left, and on the right an example comparing the optimal symbols (solid) and the local approximations we propose (dashed); see text following (3.3) for details.

2. Classical Schwarz method. Choosing in (1.4) the identity operator $\mathcal{B}_{i}=I$, $i=1,2$, gives the classical Schwarz algorithm. By linearity it suffices to consider only the case $f=0$ and analyze convergence to the zero solution. Taking a Fourier transform of the Schwarz algorithm (1.4) with $\mathcal{B}_{i}=I$ in the $\theta$ direction, we obtain

$$
\begin{aligned}
\partial_{r r} \hat{u}_{1}^{n}+\frac{1}{r} \partial_{r} \hat{u}_{1}^{n}-\left(\frac{k^{2}}{r^{2}}+\eta\right) \hat{u}_{1}^{n} & =0, & & r<R_{1}, k \in \mathbb{R}, \\
\hat{u}_{1}^{n}\left(R_{1}, k\right) & =\hat{u}_{2}^{n-1}\left(R_{1}, k\right), & & k \in \mathbb{R}, \\
\partial_{r r} \hat{u}_{2}^{n}+\frac{1}{r} \partial_{r} \hat{u}_{2}^{n}-\left(\frac{k^{2}}{r^{2}}+\eta\right) \hat{u}_{2}^{n} & =0, & & r>R_{2}, k \in \mathbb{R}, \\
\hat{u}_{2}^{n}\left(R_{2}, k\right) & =\hat{u}_{1}^{n-1}\left(R_{2}, k\right), & & k \in \mathbb{R} .
\end{aligned}
$$

Hence subdomain solutions in the Fourier transformed domain are of the form

$$
\hat{u}_{i}^{n}(r, k)=A_{i}(k) I_{k}(\sqrt{\eta} r)+B_{i}(k) K_{k}(\sqrt{\eta} r), \quad i=1,2,
$$

where $I_{k}(x)$ and $K_{k}(x)$ are the order $k$ modified Bessel functions of the first and second kinds. Since iterates need to stay bounded, we obtain for the subdomain solutions

$$
\hat{u}_{1}^{n}(r, k)=A_{1}^{n}(k) I_{k}(\sqrt{\eta} r), \quad \hat{u}_{2}^{n}(r, k)=B_{2}^{n}(k) K_{k}(\sqrt{\eta} r) .
$$

Inserting these solutions into algorithm (2.1), we obtain by induction

$$
\hat{u}_{1}^{2 n}\left(R_{2}, k\right)=\rho_{\text {cla }}^{n} \hat{u}_{1}^{0}\left(R_{2}, k\right), \quad \hat{u}_{2}^{2 n}\left(R_{1}, k\right)=\rho_{\text {cla }}^{n} \hat{u}_{2}^{0}\left(R_{1}, k\right),
$$

where the convergence factor $\rho_{c l a}(k, R, L, \eta)$ of the classical Schwarz algorithm is

$$
\rho_{c l a}(k, R, L, \eta):=\frac{I_{k}(\sqrt{\eta} R)}{K_{k}(\sqrt{\eta} R)} \cdot \frac{K_{k}(\sqrt{\eta}(R+L))}{I_{k}(\sqrt{\eta}(R+L))} \quad \forall k \in \mathbb{R} .
$$

THEOREM 2.1. The Schwarz algorithm (1.4) with classical transmission conditions $\mathcal{B}_{i}=I$ is convergent if and only if the overlap $L$ is greater than zero.

Proof. Note that $I_{k}(x)$ is exponentially increasing and $K_{k}(x)$ is exponentially decreasing in $x$ for $x>0$ (see [1]), and hence $\left|\rho_{\text {cla }}(k, R, L, \eta)\right|=\rho_{\text {cla }}(k, R, L, \eta)<1$ if and only if $L>0$.

The following lemma will often be used in the rest of the paper. 
LEMmA 2.2. Let $v_{k}(x):=\frac{K_{k}^{\prime}(x)}{K_{k}(x)}$ and $w_{k}(x):=\frac{I_{k}^{\prime}(x)}{I_{k}(x)}$. Then, for fixed $x>0$, $G_{k}(x):=-v_{k}(x)+w_{k}(x)$ is an increasing function of $k$ for $k>0$, and we have

$$
G_{k}(x)=-\frac{K_{k}^{\prime}(x)}{K_{k}(x)}+\frac{I_{k}^{\prime}(x)}{I_{k}(x)}=\frac{K_{k+1}(x)}{K_{k}(x)}+\frac{I_{k+1}(x)}{I_{k}(x)}=\frac{1}{x I_{k}(x) K_{k}(x)} .
$$

Proof. The first result is obtained directly from Lemma A.3 in the appendix. For the second result, the first equation is just the definition, the second is obtained by applying the recurrence relation 9.6.26 in [1], and the last one is obtained by applying the Wronskian 9.6.15 in [1].

THEOREM 2.3. The asymptotic convergence factor of the classical Schwarz method as $L \rightarrow 0$ is

$$
\max _{k \geq k_{\text {min }}}\left|\rho_{c l a}(k, L, R, \eta)\right|=1-G_{\min } \sqrt{\eta} L+O\left(L^{2}\right)
$$

where $G_{\text {min }}=G_{k_{\text {min }}}(\sqrt{\eta} R)$ and $k_{\text {min }}$ is the estimate of the lowest frequency involved in the computation.

Proof. A Taylor expansion of the convergence factor for $L$ small gives

$$
\begin{aligned}
\rho_{c l a}(k, L, R, \eta) & =1-\frac{K_{k}^{\prime}(\sqrt{\eta} R) I_{k}(\sqrt{\eta} R)-I_{k}^{\prime}(\sqrt{\eta} R) K_{k}(\sqrt{\eta} R)}{I_{k}(\sqrt{\eta} R) K_{k}(\sqrt{\eta} R)} \sqrt{\eta} L+O\left(L^{2}\right) \\
& =1-G_{k}(\sqrt{\eta} R) \sqrt{\eta} L+O\left(L^{2}\right) .
\end{aligned}
$$

Now using the monotonicity of $G_{k}(x)$ in $k$ for $x>0$ fixed we get (2.7).

Remark 2.4. In the circular domain decomposition described in (1.2), the interface is a circle, which is equivalent to imposing periodic boundary conditions at $\theta=0$ and $2 \pi$. Then the lowest possible frequency on these domains would be $k_{\min }=1$.

We now compare the results above for the circular domain decomposition to the results in [17] for the straight interface, where the classical Schwarz method has an asymptotic convergence factor of $1-2 \sqrt{\eta} L+O\left(L^{2}\right)$ as $L \rightarrow 0$. We thus need to study the relation between 2 and $G_{\min }$. If $k_{\min } \neq 0$, by 9.6.7 and 9.6.9 in [1] we obtain $G_{\text {min }} \sim \frac{2 k_{\min }}{R}$ as $R \rightarrow 0$. Therefore, when $R$ is small, we have $G_{\min }>2$. When $R \rightarrow \infty$, we obtain by 9.7 .5 in [1]

$$
G_{\text {min }} \sim 2\left(1-\frac{1}{2} \frac{4 k_{\min }^{2}-1}{(2 \sqrt{\eta} R)^{2}}+\frac{1}{2} \frac{3}{4} \frac{\left(4 k_{\min }^{2}-1\right)\left(4 k_{\min }^{2}-9\right)}{(2 \sqrt{\eta} R)^{4}}-\cdots\right)^{-1} \sim 2,
$$

which is consistent with the straight interface analysis. If $k_{\min }=0$, we first analyze if $\lim _{x \rightarrow 0} \frac{1}{x I_{0}(x) K_{0}(x)}>2$ holds. From the expression of $I_{0}(x)$ and $K_{0}(x)$ in 9.6.12 and 9.6.13 in [1], we have $\lim _{x \rightarrow 0} x I_{0}(x) K_{0}(x)=0$, and thus when $R$ is small we have $G_{0}(\sqrt{\eta} R)>2$. On the other hand, $\lim _{x \rightarrow \infty} G_{0}(x)=2$, since from 9.6 .12 and 9.6.13 in [1] we obtain $\lim _{x \rightarrow \infty} \frac{I_{1}(x)}{I_{0}(x)}=1$ and $\lim _{x \rightarrow \infty} \frac{K_{1}(x)}{K_{0}(x)}=1$. We illustrate these comparisons in Figure 2 (left). In summary, if $R$ is small, then the classical Schwarz method with circular domain decomposition converges faster than with a straight interface domain decomposition. When $R$ is large, both perform similarly.

3. Optimized Schwarz algorithms. Taking $\mathcal{B}_{i}(u)=\partial_{r} u+\mathcal{S}_{i} u$ in (1.4) leads to the optimized Schwarz algorithm

$$
\begin{aligned}
\partial_{r r} u_{1}^{n}+\frac{1}{r} \partial_{r} u_{1}^{n}+\frac{1}{r^{2}} \partial_{\theta \theta} u_{1}^{n}-\eta u_{1}^{n} & =f & & \text { in } \Omega_{1}, \\
\left(\partial_{r}+\mathcal{S}_{1}\right) u_{1}^{n}\left(R_{1}, \theta\right) & =\left(\partial_{r}+\mathcal{S}_{1}\right) u_{2}^{n-1}\left(R_{1}, \theta\right) & & \theta \in[0,2 \pi), \\
\partial_{r r} u_{2}^{n}+\frac{1}{r} \partial_{r} u_{2}^{n}+\frac{1}{r^{2}} \partial_{\theta \theta} u_{2}^{n}-\eta u_{2}^{n} & =f & & \text { in } \Omega_{2}, \\
\left(\partial_{r}+\mathcal{S}_{2}\right) u_{2}^{n}\left(R_{2}, \theta\right) & =\left(\partial_{r}+\mathcal{S}_{2}\right) u_{1}^{n-1}\left(R_{2}, \theta\right) & & \theta \in[0,2 \pi),
\end{aligned}
$$



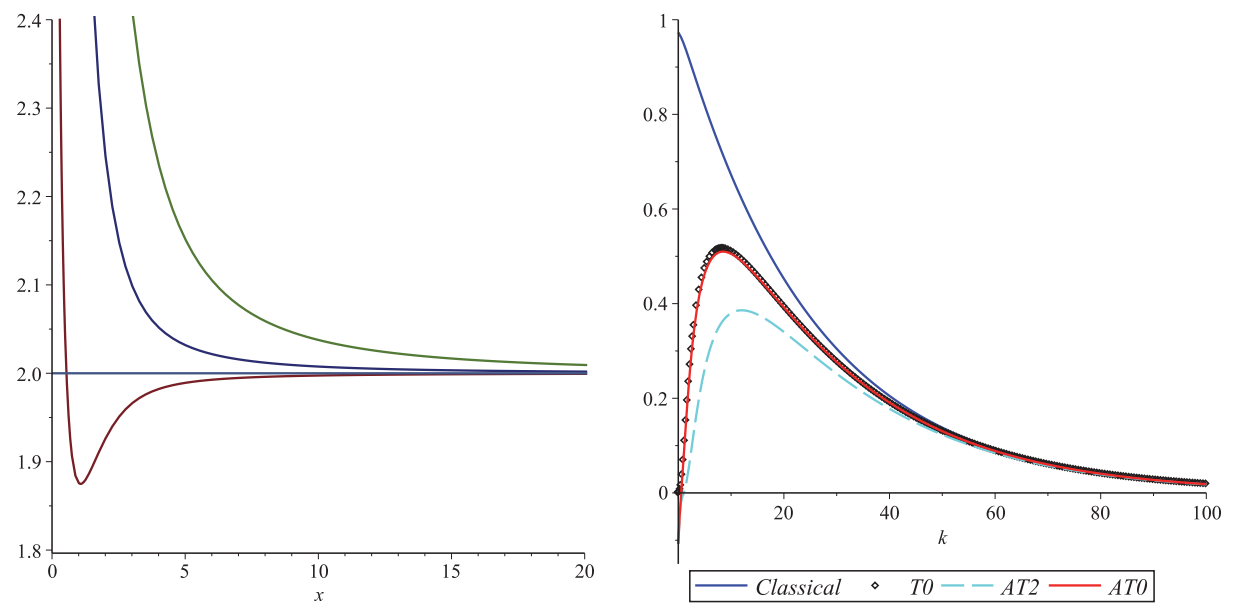

FIG. 2. Left: $G_{0}(x)$ (bottom), $G_{1}(x)$ (middle), and $G_{2}(x)$ (top) compared to 2. Right: Convergence factors for the Schwarz method with classical, T0, AT0, and AT2 transmission conditions for $\eta=2, R=0.5, L=0.01$.

where $\mathcal{S}_{i}, i=1,2$, are linear operators along the interface in the $\theta$ direction that will be determined to get the best possible performance. Setting $f=0$, taking a Fourier transform in the $\theta$ direction, and denoting by $\sigma_{i}(k)$ the Fourier symbols of the operators $\mathcal{S}_{i}, i=1,2$, we find after a short calculation the convergence factor of (3.1) to be

$$
\rho_{\text {opt }}\left(k, L, R, \eta, \sigma_{1}, \sigma_{2}\right)=\frac{\sqrt{\eta} v_{k}(\sqrt{\eta}(R+L))+\sigma_{1}(k)}{\sqrt{\eta} w_{k}(\sqrt{\eta}(R+L))+\sigma_{1}(k)} \cdot \frac{\sqrt{\eta} w_{k}(\sqrt{\eta} R)+\sigma_{2}(k)}{\sqrt{\eta} v_{k}(\sqrt{\eta} R)+\sigma_{2}(k)} \cdot \rho_{c l a}(k, L, R, \eta),
$$

where $v_{k}(x)$ and $w_{k}(x)$ are as defined in Lemma 2.2.

THEOREM 3.1 (optimal Schwarz method). If $\sigma_{1}(k)=-\sqrt{\eta} v_{k}(\sqrt{\eta}(R+L))$ and $\sigma_{2}(k)=-\sqrt{\eta} w_{k}(\sqrt{\eta} R)$, then the new Schwarz algorithm (3.1) will converge in two iterations.

Proof. The proof is similar to the proof of Lemma 2.2 in [15].

Unfortunately, the optimal choice of $\sigma_{i}(k), i=1,2$, in Theorem 3.1 results in nonlocal operators $\mathcal{S}_{i}, i=1,2$, which are hard to implement and expensive to use in practice. If we choose for $\sigma_{i}(k), i=1,2$, however, polynomials in $k$, we obtain local transmission conditions involving derivatives w.r.t. $\theta$. Here, we choose polynomials of degree two,

$$
\sigma_{1}^{a p p}(k)=p_{1}+q_{1} k^{2}, \quad \sigma_{2}^{a p p}(k)=-p_{2}-q_{2} k^{2},
$$

since second order derivatives corresponding to $k^{2}$ are already contained in the differential operator $\Delta-\eta$, and thus the transmission conditions will not increase the bandwidth of the discretized problem. We thus approximate by (3.3) the best choice in Theorem 3.1, which contains the ratios of the modified Bessel functions and their derivatives in $v_{k}$ and $w_{k}$. We show in Figure 1 (right) an example comparing the optimal symbols with the second order approximations we propose. The transmission conditions resulting from the choice (3.3) are

$$
\mathcal{B}_{1}=\partial_{r}+p_{1}-q_{1} \partial_{\theta \theta}, \quad \mathcal{B}_{2}=\partial_{r}-p_{2}+q_{2} \partial_{\theta \theta} .
$$



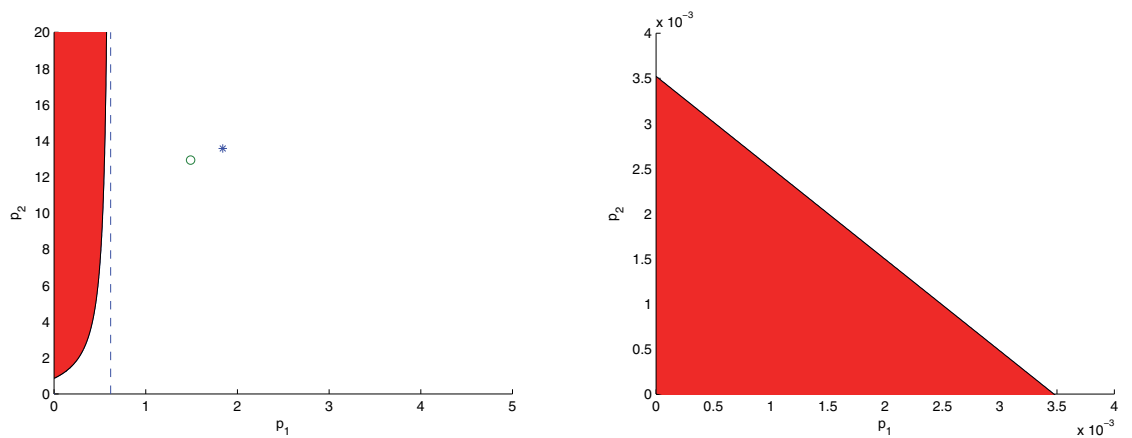

FIG. 3. Domain in which the optimized Schwarz method with 2-sided Robin condition converges for $\eta=2, R=0.5$, and $L=0.01$. On the left, the parameters outside the shaded region lead to a convergent method (the vertical dashed line is the curve's asymptote). On the right we show a zoom of the left close to zero, where again we can show convergence outside the shaded region. The star indicates the position of the optimized parameters from Theorem 3.13, and the circle that obtained by equioscillation.

Inserting the expression (3.3) of $\sigma_{i}(k)$ into (3.2), the convergence factor of the parallel Schwarz algorithm (3.1) becomes

$$
\begin{aligned}
& \rho\left(k, L, R, \eta, p_{1}, p_{2}, q_{1}, q_{2}\right) \\
= & \frac{-\sqrt{\eta} v_{k}(\sqrt{\eta}(R+L))-p_{1}-q_{1} k^{2}}{\sqrt{\eta} w_{k}(\sqrt{\eta}(R+L))+p_{1}+q_{1} k^{2}} \cdot \frac{\sqrt{\eta} w_{k}(\sqrt{\eta} R)-p_{2}-q_{2} k^{2}}{-\sqrt{\eta} v_{k}(\sqrt{\eta} R)+p_{2}+q_{2} k^{2}} \cdot \rho_{\text {cla }}(k, L, R, \eta) .
\end{aligned}
$$

Remark 3.2. When the domain $\Omega$ is decomposed into two half planes, the optimized Schwarz method for problem (1.1) converges for all $p_{i}>0$ and $q_{i} \geq 0$; see [17]. However, this is not true for a circular domain decomposition. For example, the choice of $p_{1}=0.01, p_{2}=100, q_{1}=q_{2}=0$ leads to a nonconvergent parallel Schwarz method with overlap $L=0.01$ for the model problem with parameter $\eta=2$ and the circular domain decomposition with $R=0.5$ : the convergence factor equals -1.1583 at $k=1$. Fortunately, in the set $\left\{p_{i}>0, q_{i} \geq 0, i=1,2\right\}$, the measure of the set in which the parameters result in a nonconvergent Schwarz method is quite small. In Figure 3, we plot the region where the parameters result in convergent optimized Schwarz methods.

The goal of optimized Schwarz methods is to choose the free parameters $p_{i}>0$, $q_{i} \geq 0, i=1,2$, to obtain good performance. The best performance is obtained by minimizing the convergence factor over all frequencies relevant to the problem, i.e., solving

$$
\min _{p_{i}>0, q_{i} \geq 0}\left(\max _{k \geq k_{\text {min }}}\left|\rho\left(k, L, R, \eta, p_{1}, p_{2}, q_{1}, q_{2}\right)\right|\right) .
$$

However, the modified Bessel functions $I_{k}(x)$ and $K_{k}(x)$ in the convergence factor depend on $k$ in a complicated fashion, and problem (3.6) cannot be solved directly. We therefore propose the approximation

$$
\rho_{a p p}\left(k, L, R, \eta, p_{1}, p_{2}, q_{1}, q_{2}\right)=\frac{\sqrt{\eta+\frac{k^{2}}{R^{2}}}-p_{1}-q_{1} k^{2}}{\sqrt{\eta+\frac{k^{2}}{R^{2}}}+p_{1}+q_{1} k^{2}} \cdot \frac{\sqrt{\eta+\frac{k^{2}}{R^{2}}}-p_{2}-q_{2} k^{2}}{\sqrt{\eta+\frac{k^{2}}{R^{2}}}+p_{2}+q_{2} k^{2}} e^{-2 \sqrt{\eta+\frac{k^{2}}{R^{2}}} L},
$$

which is justified by the following lemma.

Lemma 3.3 (approximation of the convergence factor). For $k>1$, there exists a 
constant $C$ independent of $k$ and $L$ such that

$$
\left|\rho\left(k, L, R, \eta, p_{1}, p_{2}, q_{1}, q_{2}\right)-\rho_{a p p}\left(k, L, R, \eta, p_{1}, p_{2}, q_{1}, q_{2}\right)\right| \leq C\left(\frac{1}{k^{2}}+\frac{L}{k}+L^{2}\right) .
$$

Proof. For the modified Bessel functions, the so-called Turán inequalities [4] hold,

$$
\begin{gathered}
\sqrt{\frac{k}{k+1} x^{2}+k^{2}}<\frac{x I_{k}^{\prime}(x)}{I_{k}(x)}<\sqrt{x^{2}+k^{2}} \forall x>0, k>0, \\
-\sqrt{\frac{k}{k-1} x^{2}+k^{2}}<\frac{x K_{k}^{\prime}(x)}{K_{k}(x)}<-\sqrt{x^{2}+k^{2}} \forall x>0, k>1,
\end{gathered}
$$

and we thus have for $k>1$ and $x>0$

$$
\sqrt{\frac{k}{k+1}+\frac{k^{2}}{x^{2}}}<w_{k}(x)<\sqrt{1+\frac{k^{2}}{x^{2}}}<-v_{k}(x)<\sqrt{\frac{k}{k-1}+\frac{k^{2}}{x^{2}}} .
$$

Denoting

$$
\tilde{\rho}\left(k, L, R, \eta, p_{1}, p_{2}, q_{1}, q_{2}\right)=\frac{-\sqrt{\eta} v_{k}(\sqrt{\eta}(R+L))-p_{1}-q_{1} k^{2}}{\sqrt{\eta} w_{k}(\sqrt{\eta}(R+L))+p_{1}+q_{1} k^{2}} \cdot \frac{\sqrt{\eta} w_{k}(\sqrt{\eta} R)-p_{2}-q_{2} k^{2}}{-\sqrt{\eta} v_{k}(\sqrt{\eta} R)+p_{2}+q_{2} k^{2}},
$$

we obtain $\rho=\tilde{\rho} \cdot \rho_{c l a}$, and thus first estimate $\tilde{\rho}$. If $p_{1}+q_{1} k^{2}<-\sqrt{\eta} v_{k}(\sqrt{\eta}(R+L))$ and $p_{2}+q_{2} k^{2}<\sqrt{\eta} w_{k}(\sqrt{\eta} R)$, we obtain using (3.11) that

$$
\begin{aligned}
\tilde{\rho} & \leq \frac{\sqrt{\frac{k \eta}{k-1}+\left(\frac{k}{R+L}\right)^{2}}-p_{1}-q_{1} k^{2}}{\sqrt{\frac{k \eta}{k+1}+\left(\frac{k}{R+L}\right)^{2}}+p_{1}+q_{1} k^{2}} \cdot \frac{\sqrt{\eta+\frac{k^{2}}{R^{2}}}-p_{2}-q_{2} k^{2}}{\sqrt{\eta+\frac{k^{2}}{R^{2}}}+p_{2}+q_{2} k^{2}} \\
& =\frac{\sqrt{\eta+\frac{k^{2}}{R^{2}}}-p_{1}-q_{1} k^{2}}{\sqrt{\eta+\frac{k^{2}}{R^{2}}}+p_{1}+q_{1} k^{2}} \cdot \frac{\sqrt{\eta+\frac{k^{2}}{R^{2}}}-p_{2}-q_{2} k^{2}}{\sqrt{\eta+\frac{k^{2}}{R^{2}}}+p_{2}+q_{2} k^{2}}+O\left(\frac{L}{k}+\frac{1}{k^{3}}\right)
\end{aligned}
$$

In addition, we have

$$
\begin{aligned}
\tilde{\rho} & \geq \frac{\sqrt{\eta+\left(\frac{k}{R+L}\right)^{2}}-p_{1}-q_{1} k^{2}}{\sqrt{\eta+\left(\frac{k}{R+L}\right)^{2}}+p_{1}+q_{1} k^{2}} \cdot \frac{\sqrt{\frac{k \eta}{k+1}+\frac{k^{2}}{R^{2}}}-p_{2}-q_{2} k^{2}}{\sqrt{\frac{k \eta}{k-1}+\frac{k^{2}}{R^{2}}}+p_{2}+q_{2} k^{2}} \\
& =\frac{\sqrt{\eta+\frac{k^{2}}{R^{2}}}-p_{1}-q_{1} k^{2}}{\sqrt{\eta+\frac{k^{2}}{R^{2}}}+p_{1}+q_{1} k^{2}} \cdot \frac{\sqrt{\eta+\frac{k^{2}}{R^{2}}}-p_{2}-q_{2} k^{2}}{\sqrt{\eta+\frac{k^{2}}{R^{2}}}+p_{2}+q_{2} k^{2}}+O\left(\frac{L}{k}+\frac{1}{k^{3}}\right) .
\end{aligned}
$$

Noting that

$$
\begin{aligned}
\rho_{c l a}(k, L, R, \eta) & =1+\frac{K_{k}^{\prime}(\sqrt{\eta} R) I_{k}(\sqrt{\eta} R)-I_{k}^{\prime}(\sqrt{\eta} R) K_{k}(\sqrt{\eta} R)}{I_{k}(\sqrt{\eta} R) K_{k}(\sqrt{\eta} R)} \sqrt{\eta} L+O\left(L^{2}\right) \\
& =1-2 \sqrt{\eta+\frac{k^{2}}{R^{2}}} L+O\left(\frac{1}{k^{2}}+L^{2}\right) \\
& =\exp \left(-2 \sqrt{\eta+\frac{k^{2}}{R^{2}}} L\right)+O\left(\frac{1}{k^{2}}+\frac{L}{k}+L^{2}\right),
\end{aligned}
$$

we obtain that, if $p_{1}+q_{1} k^{2}<-\sqrt{\eta} v_{k}(\sqrt{\eta}(R+L))$ and $p_{2}+q_{2} k^{2}<\sqrt{\eta} w_{k}(\sqrt{\eta} R)$, then there exists a constant $C$ independent of $k$ and $L$ such that (3.8) holds. Since the Turán-type inequalities (3.9) and (3.10) hold for any $k>1, x>0$, similar results can be obtained for the other cases. 
3.1. Low-frequency approximations. From the proof of Theorem 2.3 we see that $\rho_{\text {cla }}(k, L, R, \eta)=1+G_{k}(\sqrt{\eta} R) \sqrt{\eta} L+O\left(L^{2}\right)$, and together with Lemma 2.2 we conclude that $\rho_{\text {cla }}(k, L, R, \eta)$ decreases in $k$ if $L$ is small, as shown in Figure 2 (right). This shows that the classical Schwarz method cannot eliminate low-frequency errors efficiently. One can therefore use the transmission conditions that are efficient for low frequencies by using a Taylor expansion about $k=0$ of the optimal symbols $\sigma_{1}(k)=-\sqrt{\eta} v_{k}(\sqrt{\eta}(R+L))$ and $\sigma_{2}(k)=-\sqrt{\eta} w_{k}(\sqrt{\eta} R)$.

Using the identities $I_{0}^{\prime}(x)=I_{1}(x)$ and $K_{0}^{\prime}(x)=-K_{1}(x)$ [1], we obtain

$$
\sigma_{1}(k)=\sqrt{\eta} \frac{K_{1}(\sqrt{\eta}(R+L))}{K_{0}(\sqrt{\eta}(R+L))}+O(k), \quad \sigma_{2}(k)=-\sqrt{\eta} \frac{I_{1}(\sqrt{\eta} R)}{I_{0}(\sqrt{\eta} R)}+O(k) .
$$

Therefore, with the choices of $p_{1}=\sqrt{\eta} \frac{K_{1}(\sqrt{\eta}(R+L))}{K_{0}(\sqrt{\eta}(R+L))}$ and $p_{2}=\sqrt{\eta} \frac{I_{1}(\sqrt{\eta} R)}{I_{0}(\sqrt{\eta} R)}$, we obtain a Schwarz algorithm with the convergence factor

$$
\begin{aligned}
\rho_{T 0}(k, L, R, \eta)= & \frac{-\sqrt{\eta} K_{k}^{\prime}(\sqrt{\eta}(R+L))-\sqrt{\eta} \frac{K_{1}(\sqrt{\eta}(R+L))}{K_{0}(\sqrt{\eta}(R+L))} K_{k}(\sqrt{\eta}(R+L))}{\sqrt{\eta} I_{k}^{\prime}(\sqrt{\eta}(R+L))+\sqrt{\eta} \frac{K_{1}(\sqrt{\eta}(R+L))}{K_{0}(\sqrt{\eta}(R+L)} I_{k}(\sqrt{\eta}(R+L))} \\
& \cdot \frac{\sqrt{\eta} I_{k}^{\prime}(\sqrt{\eta} R)-\sqrt{\eta} \frac{I_{1}(\sqrt{\eta} R)}{I_{0}(\sqrt{\eta} R)} I_{k}(\sqrt{\eta} R)}{\sqrt{\eta} K_{k}^{\prime}(\sqrt{\eta} R)+\sqrt{\eta} \frac{I_{1}(\sqrt{\eta}) R}{I_{0}(\sqrt{\eta} \eta)} K_{k}(\sqrt{\eta} R)} .
\end{aligned}
$$

Theorem 3.4 (Taylor asymptotics). As $L \rightarrow 0$, the asymptotic convergence factor of the optimized Schwarz method with Taylor transmission conditions of order 0 satisfies

$$
\max _{|k|}\left|\rho_{T 0}(k, L, R, \eta)\right|=1-4 \eta^{\frac{1}{4}} G_{0}^{\frac{1}{2}}(\sqrt{\eta} R) L^{\frac{1}{2}}+O(L) .
$$

Proof. We first show that $\rho_{\text {app }}\left(k, L, R, \eta, \sqrt{\eta} \frac{K_{1}(\sqrt{\eta}(R+L))}{K_{0}(\sqrt{\eta}(R+L))}, \sqrt{\eta} \frac{I_{1}(\sqrt{\eta} R)}{I_{0}(\sqrt{\eta} R)}, 0,0\right)$ attains its unique maximum in the interior asymptotically at $k_{T 0}=\eta^{\frac{1}{4}} R G_{0}^{\frac{1}{2}}(\sqrt{\eta} R) L^{-\frac{1}{2}}$. To do so, we solve the derivative of $\rho_{a p p}\left(k, L, R, \eta, p_{1}, p_{2}, 0,0\right)$ in $k$ with $p_{1}=\sqrt{\eta} \frac{K_{1}(\sqrt{\eta}(R+L))}{K_{0}(\sqrt{\eta}(R+L))}$ and $p_{2}=\sqrt{\eta} \frac{I_{1}(\sqrt{\eta} R)}{I_{0}(\sqrt{\eta} R)}$ and find the positive solution to be

$$
k_{T 0}=\frac{\sqrt{2} R}{2} \sqrt{\frac{L p_{1}^{2}+L p_{2}^{2}-2 L \eta+p_{2}+p_{1}+\sqrt{\Phi}}{L}},
$$

where $\Phi=p_{2}^{2}+p_{1}^{2}+2 p_{1} p_{2}-2 L^{2} p_{1}^{2} p_{2}^{2}-2 L p_{1}^{2} p_{2}-2 L p_{2}^{2} p_{1}+L^{2} p_{1}^{4}+2 L p_{1}^{3}+L^{2} p_{2}^{4}+2 L p_{2}^{3}$. Although this expression is too complicated to be used directly, it tells us that $k_{T 0}$ behaves asymptotically like $k_{T 0}=C_{k} L^{-\frac{1}{2}}$, where $C_{k}$ is a constant to be determined. Inserting this into the derivative of $\rho_{a p p}\left(k, L, R, \eta, \sqrt{\eta} \frac{K_{1}(\sqrt{\eta}(R+L))}{K_{0}(\sqrt{\eta}(R+L))}, \sqrt{\eta} \frac{I_{1}(\sqrt{\eta} R)}{I_{0}(\sqrt{\eta} R)}, 0,0\right)$ and expanding for $L$ small, we obtain

$$
\rho_{\text {app }}^{\prime}\left(k, L, R, \eta, \sqrt{\eta} \frac{K_{1}(\sqrt{\eta}(R+L))}{K_{0}(\sqrt{\eta}(R+L))}, \sqrt{\eta} \frac{I_{1}(\sqrt{\eta} R)}{I_{0}(\sqrt{\eta} R)}, 0,0\right)=2\left(\frac{\sqrt{\eta} R G_{0}(\sqrt{\eta} R)}{C_{k}^{2}}-\frac{1}{R}\right) L+O\left(L^{2}\right) .
$$

The constant $C_{k}$ is thus obtained by setting the leading term to zero, and $k_{T 0}$ follows. Using Lemma 3.3, we then obtain

$$
\begin{aligned}
& \rho_{T 0}\left(k_{T 0}, L, R, \eta\right) \\
= & \rho_{a p p}\left(k_{T 0}, L, R, \eta, \sqrt{\eta} \frac{K_{1}(\sqrt{\eta}(R+L))}{K_{0}(\sqrt{\eta}(R+L))}, \sqrt{\eta} \frac{K_{1}(\sqrt{\eta}(R+L))}{K_{0}(\sqrt{\eta}(R+L))}, 0,0\right)+O\left(\frac{1}{k_{T 0}^{2}}+\frac{L}{k_{T 0}}+L^{2}\right) \\
= & 1-4 \eta^{\frac{1}{4}} G_{0}^{\frac{1}{2}}(\sqrt{\eta} R) L^{\frac{1}{2}}+O(L) .
\end{aligned}
$$


Higher order Taylor conditions could in principle be obtained, expanding the optimal choices of $\sigma_{1}(k)$ and $\sigma_{2}(k)$ in $k$ to higher orders, but the derivatives of the modified Bessel functions $I_{k}(x)$ and $K_{k}(x)$ with respect to $k$ are too complicated to be of use for further analysis [1], and one could only exploit them numerically. We therefore propose as an alternative using expansions of the approximate optimal choice $\sigma_{1}^{a p p}(k)=\sqrt{\eta+\frac{k^{2}}{R_{1}^{2}}}, \sigma_{2}^{a p p}(k)=-\sqrt{\eta+\frac{k^{2}}{R_{2}^{2}}}$ we found based on the Turán-type inequalities. From

$$
\sqrt{\eta+\frac{k^{2}}{R_{i}^{2}}}=\sqrt{\eta}+\frac{k^{2}}{2 \sqrt{\eta} R_{i}}+O\left(k^{4}\right),
$$

a zeroth order approximation is then given by $p_{1}=p_{2}=\sqrt{\eta}, q_{1}=q_{2}=0$, while a second order approximation is given by $p_{i}=\sqrt{\eta}, q_{i}=\frac{1}{2 \sqrt{\eta} R_{i}}, i=1,2$. These choices lead to the Schwarz method with approximate Taylor transmission condition of order 0 (AT0) and order 2 (AT2) and corresponding convergence factors

$$
\rho_{A T 0}(k, L, R, \eta)=\frac{-\sqrt{\eta} K_{k}^{\prime}(\sqrt{\eta}(R+L))-\sqrt{\eta} K_{k}(\sqrt{\eta}(R+L))}{\sqrt{\eta} I_{k}^{\prime}(\sqrt{\eta}(R+L))+\sqrt{\eta} I_{k}(\sqrt{\eta}(R+L))} \cdot \frac{\sqrt{\eta} I_{k}^{\prime}(\sqrt{\eta} R)-\sqrt{\eta} I_{k}(\sqrt{\eta} R)}{-\sqrt{\eta} K_{k}^{\prime}(\sqrt{\eta} R)+\sqrt{\eta} K_{k}(\sqrt{\eta} R)}
$$

and

$$
\begin{aligned}
\rho_{A T 2}(k, L, R, \eta)= & \frac{-\sqrt{\eta} K_{k}^{\prime}(\sqrt{\eta}(R+L))-\left(\sqrt{\eta}+\frac{k^{2}}{2 \sqrt{\eta}(R+L)}\right) K_{k}(\sqrt{\eta}(R+L))}{\sqrt{\eta} I_{k}^{\prime}(\sqrt{\eta}(R+L))+\left(\sqrt{\eta}+\frac{k^{2}}{2 \sqrt{\eta}(R+L)}\right) I_{k}(\sqrt{\eta}(R+L))} \\
& \frac{\sqrt{\eta} I_{k}^{\prime}(\sqrt{\eta} R)-\left(\sqrt{\eta}+\frac{k^{2}}{2 \sqrt{\eta} R}\right) I_{k}(\sqrt{\eta} R)}{-\sqrt{\eta} K_{k}^{\prime}(\sqrt{\eta} R)+\left(\sqrt{\eta}+\frac{k^{2}}{2 \sqrt{\eta} R}\right) K_{k}(\sqrt{\eta} R)} .
\end{aligned}
$$

These convergence factors are shown in Figure 2 (right), and we see that AT0 is as efficient as T0, and AT2 is better than T0.

TheOREM 3.5 (approximate Taylor asymptotics). For $L \rightarrow 0$, the asymptotic convergence factors of the optimized Schwarz methods with approximate Taylor transmission conditions of order 0 and 2 satisfy

$$
\begin{aligned}
& \max _{|k|}\left|\rho_{A T 0}(k, L, R, \eta)\right|=1-4 \sqrt{2} \eta^{\frac{1}{4}} \sqrt{L}+O(L), \\
& \max _{|k|}\left|\rho_{A T 2}(k, L, R, \eta)\right|=1-8 \eta^{\frac{1}{4}} \sqrt{L}+O(L) .
\end{aligned}
$$

Proof. A direct calculation shows that $\rho_{a p p}(k, L, R, \eta, \sqrt{\eta}, \sqrt{\eta}, 0,0)$ attains its unique interior maximum asymptotically at $k_{A T 0}=\sqrt{2} \eta^{\frac{1}{4}} R L^{-\frac{1}{2}}$, which gives with Lemma 3.3

$$
\begin{aligned}
\rho_{A T 0}\left(k_{A T 0}, L, R, \eta\right) & =\rho_{a p p}\left(k_{A T 0}, L, R, \eta, \sqrt{\eta}, \sqrt{\eta}, 0,0\right)+O\left(\frac{1}{k_{A T 0}^{2}}+\frac{L}{k_{A T 0}}+L^{2}\right) \\
& =1-4 \sqrt{2} \eta^{\frac{1}{4}} \sqrt{L}+O(L) .
\end{aligned}
$$

Similarly, we obtain that $\rho_{a p p}\left(k, L, R, \eta, \sqrt{\eta}, \sqrt{\eta}, \frac{1}{2 \sqrt{\eta}(R+L)}, \frac{1}{2 \sqrt{\eta} R}\right)$ attains its unique interior maximum, asymptotically at $k_{A T 2}=2 \eta^{\frac{1}{4}} R L^{-\frac{1}{2}}$, which leads with Lemma 3.3 to

$$
\begin{aligned}
\rho_{A T 2}\left(k_{A T 2}, L, R, \eta\right) & =\rho_{\text {app }}\left(k_{A T 2}, L, R, \eta, \sqrt{\eta}, \sqrt{\eta}, \frac{1}{2 \sqrt{\eta}(R+L)}, \frac{1}{2 \sqrt{\eta} R}\right)+O\left(\frac{1}{k_{A T 2}^{2}}+\frac{L}{k_{A T 2}}+L^{2}\right) \\
& =1-8 \eta^{\frac{1}{4}} \sqrt{L}+O(L) . \quad \square
\end{aligned}
$$


We now compare the two Taylor conditions T0 and AT0 analytically: we expand the parameters $p_{1}$ and $p_{2}$ of T0 for $R$ large and find

$$
\begin{aligned}
& p_{1}=\sqrt{\eta} \frac{K_{1}\left(\sqrt{\eta} R_{1}\right)}{K_{0}\left(\sqrt{\eta} R_{1}\right)}=\sqrt{\eta}+\frac{1}{2 R_{1}}-\frac{1}{8 \sqrt{\eta} R_{1}^{2}}+\frac{1}{8 \eta R_{1}^{3}}+O\left(\frac{1}{R_{1}^{4}}\right), \\
& p_{2}=\sqrt{\eta} \frac{I_{1}\left(\sqrt{\eta} R_{2}\right)}{I_{0}\left(\sqrt{\eta} R_{2}\right)}=\sqrt{\eta}-\frac{1}{2 R_{2}}-\frac{1}{8 \sqrt{\eta} R_{2}^{2}}-\frac{1}{8 \eta R_{2}^{3}}+O\left(\frac{1}{R_{2}^{4}}\right) .
\end{aligned}
$$

This shows that taking the first terms in the expansions of T0 leads to the AT0 conditions, which is also a transmission condition found using microlocal analysis, namely, MATC; see [5]. Taking the first two terms in the expansion above results in MATC2, and taking the first four terms leads to MATC3, two further transmission conditions based on microlocal analysis. Microlocal analysis can also be used to construct higher order transmission conditions; see [5].

From our analysis of $G_{0}(x)$ in section 2, we see that for $R$ large, the T0 conditions perform slightly worse than the AT0 conditions; however, for $R$ small, the T0 conditions perform better than the AT0 conditions. In fact, for the T0 condition we have for $R$ small

$$
p_{1}=\sqrt{\eta} \frac{K_{1}\left(\sqrt{\eta} R_{1}\right)}{K_{0}\left(\sqrt{\eta} R_{1}\right)}=\frac{1}{\ln 2-\ln \left(\sqrt{\eta} R_{1}\right)-\gamma} \frac{1}{R_{1}}+O\left(R_{1}\right),
$$

where $\gamma=0.5772156649 \ldots$ is the Euler constant, and

$$
p_{2}=\sqrt{\eta} \frac{I_{1}\left(\sqrt{\eta} R_{2}\right)}{I_{0}\left(\sqrt{\eta} R_{2}\right)}=\frac{\eta R_{2}}{2}+O\left(R_{2}^{3}\right)
$$

are quite different from $\sqrt{\eta}$, the parameter value of AT0.

Using similar arguments, one would also expect that the Taylor transmission conditions of order 2 would differ for $R$ small, depending on the subdomain, since the interface curvature is involved. When $R$ is large, however, as in the proof of Theorem 3.5, it is easy to show that if we choose the parameters in each subdomain to be the same, i.e., $p_{i}=\sqrt{\eta}, q_{i}=\frac{1}{2 \sqrt{\eta} R}$, the resulting Schwarz algorithm performs asymptotically like the one with approximate Taylor condition of order 2 .

3.2. Zeroth order optimized transmission conditions. Taking $p_{1}=p_{2}=p$, $q_{1}=q_{2}=0$ gives the simplified convergence factor

$$
\rho_{O O 0}(k, L, R, \eta, p)=\frac{-\sqrt{\eta} K_{k}^{\prime}(\sqrt{\eta}(R+L))-p K_{k}(\sqrt{\eta}(R+L))}{\sqrt{\eta} I_{k}^{\prime}(\sqrt{\eta}(R+L))+p I_{k}(\sqrt{\eta}(R+L))} \cdot \frac{\sqrt{\eta} I_{k}^{\prime}(\sqrt{\eta} R)-p I_{k}(\sqrt{\eta} R)}{-\sqrt{\eta} K_{k}^{\prime}(\sqrt{\eta} R)+p K_{k}(\sqrt{\eta} R)} .
$$

The optimized parameter $p^{*}$ is thus the solution of the min-max problem

$$
\min _{p>0}\left(\max _{k \geq k_{\min }}\left|\rho_{O O 0}(k, L, R, \eta, p)\right|\right) .
$$

We will in what follows often make use of the following lemma.

LEмma 3.6. Assume $k_{0}>0$ is the solution of $\min \left\{-\sqrt{\eta} v_{k}(\sqrt{\eta}(R+L))\right.$, $\left.\sqrt{\eta} w_{k}(\sqrt{\eta} R)\right\}=p$ with $p$ given asymptotically by $p=C_{p} L^{-\alpha}, 0<\alpha<1$; then $k_{0}$ is given asymptotically by $k_{0}=C_{p} R L^{-\alpha}$.

Proof. Because of the Turán-type inequalities (3.9) and (3.10), we need only show the result in the following four cases: 
1. $\sqrt{\eta+\frac{k^{2}}{R^{2}}}=p$ : in this case the equation is solved by $k_{0}=\sqrt{p^{2}-\eta} R=$ $C_{p} R L^{-\alpha}+O\left(L^{\alpha}\right)$.

2. $\sqrt{\frac{k}{k+1} \eta+\frac{k^{2}}{R^{2}}}=p$ : we insert the ansatz $k=C_{k} L^{-\beta}$ into the equation, square both sides, and use $p=C_{p} L^{-\alpha}$ to see that to balance higher order terms in $L$ we need $\beta=\alpha$ and $C_{k}=C_{p} R$.

3. $\sqrt{\eta+\frac{k^{2}}{(R+L)^{2}}}=p$ : in this case the equation is solved by $k_{0}=\sqrt{p^{2}-\eta}(R+$ $L)=C_{p} R L^{-\alpha}+O\left(L^{\alpha}\right)$.

4. $\sqrt{\frac{k}{k-1} \eta+\frac{k^{2}}{(R+L)^{2}}}=p$ : using an argument similar to that used before shows that the solution is asymptotically given by $k_{0}=C_{p} R L^{-\alpha}$.

Remark 3.7. In Lemma 3.6, if we replace min by max, the same result holds, with a similar proof.

TheOREm 3.8 (optimized Robin parameter). The parameter

$$
p^{*}=2^{-1} \eta^{\frac{1}{3}} G_{\min }^{\frac{2}{3}} L^{-\frac{1}{3}}
$$

is for $L$ small the asymptotic solution of the equioscillation problem

$$
\rho_{O O 0}\left(k_{\text {min }}, L, R, \eta, p^{*}\right)=\rho_{O O 0}\left(\bar{k}^{*}, L, R, \eta, p^{*}\right),
$$

where $\bar{k}^{*}=\bar{k}\left(L, R, \eta, p^{*}\right)=\frac{\sqrt{L\left(2 p^{*}+L\left(\left(p^{*}\right)^{2}-\eta\right)\right)} R}{L}=\eta^{\frac{1}{6}} G_{\text {min }}^{\frac{1}{3}} R L^{-\frac{2}{3}}+o\left(L^{-\frac{2}{3}}\right)$ is the location of the unique interior maximum of $\rho_{\text {app }}\left(k, L, R, \eta, p^{*}, p^{*}, 0,0\right)$.

Proof. Setting the derivative of $\rho_{a p p}$ with respect to $k$ to zero, we find a unique interior maximum at $\bar{k}(L, R, \eta, p)=\frac{\sqrt{L\left(2 p+L\left(p^{2}-\eta\right)\right)} R}{L}$. We then need to find the asymptotic expansion for $p^{*}$ for $L$ small from (3.19). We make the ansatz $p=C_{p} L^{-\alpha}$ for $\alpha>0$ and denote $p^{*}=C_{p}^{*} L^{-\alpha^{*}}$. We first consider the case $\alpha<1$, which gives with our ansatz asymptotically $\bar{k}(L, R, \eta, p)=\sqrt{2 C_{p}} R L^{-\frac{1+\alpha}{2}}$. Expanding the lefthand side of (3.19), we obtain

$$
1-\frac{2 \sqrt{\eta}}{C_{p}^{*}}\left(-\frac{K_{k_{\min }}^{\prime}(\sqrt{\eta} R)}{K_{k_{\min }}(\sqrt{\eta} R)}+\frac{I_{k_{\min }}^{\prime}(\sqrt{\eta} R)}{\left.I_{k_{\min }(\sqrt{\eta} R)}\right)} L^{\alpha^{*}}+o\left(L^{\alpha^{*}}\right)=1-\frac{2 \sqrt{\eta}}{C_{p}^{*}} G_{m i n} L^{\alpha^{*}}+o\left(L^{\alpha^{*}}\right),\right.
$$

and for the right-hand side the expansion is

$$
\begin{aligned}
& \rho_{O O 0}\left(\bar{k}^{*}, L, R, \eta, p^{*}\right)=\rho_{\text {app }}\left(\bar{k}^{*}, L, R, \eta, p^{*}, p^{*}, 0,0\right)+O\left(\frac{1}{\left(k^{*}\right)^{2}}+\frac{L}{k^{*}}+L^{2}\right)
\end{aligned}
$$

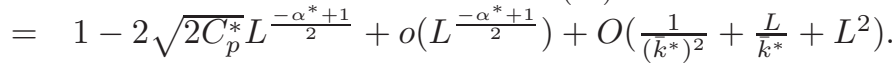

Since (3.19) holds for all $L>0$, we must have equality of the leading terms, $\frac{2 \sqrt{\eta}}{C_{p}^{*}} G_{\min }$ $L^{\alpha^{*}}=4 \sqrt{2 C_{p}^{*}} L^{\frac{-\alpha^{*}+1}{2}}$, which leads to the solution $\alpha^{*}=\frac{1}{3}, C_{p}^{*}=2^{-1} \eta^{\frac{1}{3}} G_{m i n}^{\frac{2}{3}}$, and thus the parameter $p^{*}$ in (3.18). With this asymptotic behavior of $p^{*}$, the interior maximum $\bar{k}^{*}$ behaves asymptotically like

$$
\bar{k}^{*}=\eta^{\frac{1}{6}} G_{\min }^{\frac{1}{3}} R L^{-\frac{2}{3}} .
$$

Similarly, we can also show that if $\alpha \geq 1$, there is no valid solution for $\alpha^{*}$.

THEOREM 3.9 (Robin asymptotics). The parameter value $p^{*}$ in (3.18) solves the min-max problem (3.17) asymptotically, and the convergence factor satisfies for $L$ small

$$
\max _{k \geq k_{\min }}\left|\rho_{O O 0}\left(k, L, R, \eta, p^{*}\right)\right|=1-4 \eta^{\frac{1}{6}} G_{\min }^{\frac{1}{3}} L^{\frac{1}{3}}+O\left(L^{\frac{2}{3}}\right) .
$$


Proof. We first show that $\rho_{O O 0}\left(k, L, R, \eta, p^{*}\right)$ attains its maximum asymptotically either at $k=k_{\min }$ or at $k=\bar{k}^{*}$. We denote by $\underline{k}$ the solution of $\min \left\{-\sqrt{\eta} v_{k}(\sqrt{\eta}(R+\right.$ $\left.L)), \sqrt{\eta} w_{k}(\sqrt{\eta} R)\right\}=p^{*}$, and from Lemma 3.6 we know that $\underline{k} \sim R p^{*}=2^{-1} R \eta^{\frac{1}{3}} G_{\text {min }}^{\frac{2}{3}}$ $L^{-\frac{1}{3}}$ as $L \rightarrow 0$. To show next that for $k_{\text {min }} \leq k \leq \underline{k}, \rho_{O O 0}\left(k, L, R, \eta, p^{*}\right)$ decreases in $k$, we rewrite $\rho_{O O 0}$ as $\rho_{O O 0}(k, L, R, \eta, p)=T_{1}(k, L, p) T_{2}(k, L, p) \rho_{c l a}(k, L, R, \eta)$ with $T_{1}(k, L, p):=\frac{-\sqrt{\eta} v_{k}(\sqrt{\eta}(R+L))-p}{-\sqrt{\eta} v_{k}(\sqrt{\eta} R)+p}$ and $T_{2}(k, L, p):=\frac{\sqrt{\eta} w_{k}(\sqrt{\eta} R)-p}{\sqrt{\eta} w_{k}(\sqrt{\eta}(R+L))+p}$. Expanding for $L$ small, we obtain $T_{1,2}(k, L, p)=T_{1,2}(k, 0, p)+O(L)$, and hence $T_{1,2}(k, L, p)$ increases in $k$ for $k<\underline{k}$ and $L$ small due to Theorem A.3. Since $T_{1,2}\left(k, L, p^{*}\right)$ are negative for $k<\underline{k}$, we obtain together with $\rho_{\text {cla }}(k, L, R, \eta)$ decreasing in $k$ for $k>0$ and $L$ small that $\rho_{O O 0}$, as the product of the three, decreases in $k$ for $k<\underline{k}$ and $L$ small. This implies $k=k_{\text {min }}$ is a possible maximum point for $L$ small.

Next, if $k>\underline{k}$, we know $\rho_{O O 0}\left(k, L, R, \eta, p^{*}\right)=\rho_{a p p}\left(k, L, R, \eta, p^{*}, p^{*}, 0,0\right)+O\left(L^{\frac{2}{3}}\right)$, and hence $\rho_{O O 0}\left(k, L, R, \eta, p^{*}\right)$ attains its unique interior maximum asymptotically at $\bar{k}^{*}=\eta^{\frac{1}{6}} G_{\min }^{\frac{1}{3}} R L^{-\frac{2}{3}}$.

We can now show that $p^{*}$ given by (3.18) solves the min-max problem (3.17) asymptotically. Assuming that $p=C_{p} L^{-\alpha}$, we need only show that if $\alpha \neq \alpha^{*}=\frac{1}{3}$, or $C_{p} \neq C_{p}^{*}$, we have $\max _{k}\left|\rho_{O O 0}(k, L, R, \eta, p)\right|>\max _{k}\left|\rho_{O O 0}\left(k, L, R, \eta, p^{*}\right)\right|$ for $L$ small enough. When $\alpha<\alpha^{*}$, from the proof of Theorem 3.8 we know that

$$
\begin{aligned}
\rho_{O O 0}(\bar{k}(L, R, \eta, p), L, R, \eta, p) & =\rho_{a p p}(\bar{k}(L, R, \eta, p), L, R, \eta, p, p, 0,0)+O\left(\frac{1}{k^{2}}+\frac{L}{k}+L^{2}\right) \\
& =1-2 \sqrt{2 C_{p}} L^{\frac{-\alpha+1}{2}}+o\left(L^{\frac{-\alpha+1}{2}}\right)+O\left(\frac{1}{k^{2}}+\frac{L}{k}+L^{2}\right) .
\end{aligned}
$$

Noting that $\rho_{O O 0}\left(\bar{k}\left(L, R, \eta, p^{*}\right), L, R, \eta, p^{*}\right)=1-2 \sqrt{2 C_{p}^{*} L^{\frac{-\alpha^{*}+1}{2}}}+o\left(L^{\frac{1}{3}}\right)$, we have when $L \rightarrow 0$

$$
\rho_{O O 0}(\bar{k}(L, R, \eta, p), L, R, \eta, p)>\rho_{O O 0}\left(\bar{k}\left(L, R, \eta, p^{*}\right), L, R, \eta, p^{*}\right) .
$$

When $\alpha>\alpha^{*}$, we consider the convergence factor at $k_{\min }$. In this case we have

$$
\rho_{O O 0}\left(k_{\min }, L, R, \eta, p\right)=1-2 \frac{\sqrt{\eta}}{C_{p}} G_{\min } L^{\alpha}+O\left(L^{2 \alpha}\right),
$$

which is greater than $\rho_{O O 0}\left(k_{\text {min }}, L, R, \eta, p^{*}\right)$ as $L \rightarrow 0$.

We finally consider the case $\alpha=\alpha^{*}$ but $C_{p} \neq C_{p}^{*}$. By the asymptotic expansions above we find that if $C_{p}>C_{p}^{*}$, we have $\rho_{O O 0}\left(k_{m i n}, L, R, \eta, p\right)>\rho_{O O 0}\left(k_{m i n}, L, R, \eta, p^{*}\right)$, and if $C_{p}<C_{p}^{*}$, we obtain $\rho_{O O 0}(\bar{k}(L, R, \eta, p), L, R, \eta, p)>\rho_{O O 0}\left(\bar{k}\left(L, R, \eta, p^{*}\right), L, R, \eta, p^{*}\right)$, which concludes the proof of asymptotic optimality.

To show the convergence factor estimate, we only insert $C_{p}^{*}$ and $\alpha^{*}$ into (3.21).

3.3. Second order optimized transmission condition. Setting $p_{1}=p_{2}=$ $p, q_{1}=q_{2}=q$ in the convergence factor (3.5) leads to

$$
\begin{aligned}
\rho_{O O 2}(k, L, R, \eta, p, q)= & \frac{-\sqrt{\eta} K_{k}^{\prime}(\sqrt{\eta}(R+L))-\left(p+q k^{2}\right) K_{k}(\sqrt{\eta}(R+L))}{\sqrt{\eta} I_{k}^{\prime}(\sqrt{\eta}(R+L))+\left(p+q k^{2}\right) I_{k}(\sqrt{\eta}(R+L))} \\
& \frac{\sqrt{\eta} I_{k}^{\prime}(\sqrt{\eta} R)-\left(p+q k^{2}\right) I_{k}(\sqrt{\eta} R)}{-\sqrt{\eta} K_{k}^{\prime}(\sqrt{\eta} R)+\left(p+q k^{2}\right) K_{k}(\sqrt{\eta} R)} .
\end{aligned}
$$

The optimized parameters $p^{*}$ and $q^{*}$ for the associated Schwarz algorithm thus are solutions of the min-max problem

$$
\min _{p, q>0}\left(\max _{k \geq k_{\min }}\left|\rho_{O O 2}(k, L, R, \eta, p, q)\right|\right) .
$$


To analyze this min-max problem, we need again an auxiliary result.

Lemma 3.10. Assume $\underline{k}_{2}>\underline{k}_{1}>0$ are solutions of $\min \left\{-\sqrt{\eta} v_{k}(\sqrt{\eta}(R+\right.$ $\left.L)), \sqrt{\eta} w_{k}(\sqrt{\eta} R)\right\}=p+q k^{2}$ with $p=C_{p} L^{-\alpha}$ and $q=C_{q} L^{\beta}$, where $\beta>\alpha>0$. Then $\underline{k}_{1,2}$ behave asymptotically for $L$ small like

$$
\underline{k}_{1}=C_{p} R L^{-\alpha}, \quad \underline{k}_{2}=C_{q}^{-1} R^{-1} L^{-\beta} .
$$

Proof. Using Lemma 3.6, we know that $\min \left\{-\sqrt{\eta} v_{k}(\sqrt{\eta}(R+L)), \sqrt{\eta} u_{k}(\sqrt{\eta} R)\right\}$ behaves like $\frac{k}{R}$, and the problem simplifies to solving $k^{2}-q^{-1} R^{-1} k+q^{-1} p=0$, whose solutions are $\underline{k}_{1,2}=\frac{1}{2} \frac{1 \mp \sqrt{1-4 C_{p} C_{q} R^{2} L^{\beta-\alpha}}}{C_{q} L^{\beta} R}$. Expanding for $L$ small then gives (3.24).

TheOREM 3.11 (optimized second order parameters). The parameters

$$
p^{*}=2^{-\frac{7}{5}} \eta^{\frac{2}{5}} G_{\min }^{\frac{4}{5}} L^{-\frac{1}{5}}, \quad q^{*}=2^{\frac{1}{5}} \eta^{-\frac{1}{5}} G_{\min }^{-\frac{2}{5}} R^{-2} L^{\frac{3}{5}}
$$

solve for $L$ small the equioscillation equations

$$
\begin{aligned}
\rho_{\mathrm{OO} 2}\left(k_{\text {min }}, L, R, \eta, p^{*}, q^{*}\right) & =\rho_{O O 2}\left(\bar{k}_{1}^{*}, L, R, \eta, p^{*}, q^{*}\right) \\
& =\rho_{O O 2}\left(\bar{k}_{2}^{*}, L, R, \eta, p^{*}, q^{*}\right),
\end{aligned}
$$

where $\bar{k}_{1,2}^{*}=\bar{k}_{1,2}\left(L, R, \eta, p^{*}, q^{*}\right)$ with $\bar{k}_{1,2}(L, R, \eta, p, q)=\frac{\sqrt{2}}{2 q R} \sqrt{\frac{L+2 q R^{2}-2 L p q R^{2} \mp \sqrt{\Psi}}{L}}$, and $\Psi=L^{2}+4 L q R^{2}-4 L^{2} p q R^{2}+4 q^{2} R^{4}-16 L p q^{2} R^{4}+16 L q^{3} R^{6} \eta+4 L^{2} q^{2} R^{4} \eta$, at which $\rho_{\mathrm{OO} 2}\left(k, L, R, \eta, p^{*}, q^{*}\right)$ attains its maxima asymptotically, and we have $\bar{k}_{1}^{*} \sim$ $2^{-\frac{4}{5}} \eta^{\frac{3}{10}} G_{\min }^{\frac{3}{5}} R L^{-\frac{2}{5}}, \bar{k}_{2}^{*} \sim 2^{\frac{2}{5}} \eta^{\frac{1}{10}} G_{\min }^{\frac{1}{5}} R L^{-\frac{4}{5}}$.

Proof. From the derivative of $\rho_{a p p}(k, L, R, \eta, p, p, q, q)$ in $k$ we get $\bar{k}_{1,2}$ by direct calculation. Inserting the ansatz $p=C_{p} L^{-\frac{1}{5}}$ and $q=C_{q} L^{\frac{3}{5}}$ into $\bar{k}_{1,2}$ and expanding for $L$ small, we obtain $k_{1}(L, R, \eta, p, q) \sim \sqrt{\frac{C_{p}}{C_{q}}} L^{-\frac{2}{5}}$ and $\bar{k}_{2}(L, \eta, p, q) \sim \sqrt{\frac{2}{C_{q}}} L^{-\frac{4}{5}}$. Using the same ansatz also in $\rho_{O O 2}\left(k_{m i n}, L, R, \eta, p, q\right)$ and expanding for $L$ small leads to

$$
\begin{aligned}
\rho_{O O 2}\left(k_{\min }, L, R, \eta, p, q\right) & =1-2 \eta^{\frac{1}{2}} \frac{G_{\min }}{C_{p}} L^{\frac{1}{5}}+O\left(L^{\frac{2}{5}}\right), \\
\rho_{O O 2}\left(\bar{k}_{1}, L, R, \eta, p, q\right) & =\rho_{\text {app }}\left(\bar{k}_{1}(L, R, \eta, p, q), L, R, \eta, p, p, q, q\right)+O\left(L^{\frac{8}{5}}\right) \\
& =1-8 \sqrt{C_{q} C_{p}} R L^{\frac{1}{5}}+O\left(L^{\frac{2}{5}}\right), \\
\rho_{O O 2}\left(\bar{k}_{2}, L, R, \eta, p, q\right) & =\rho_{a p p}\left(\bar{k}_{2}(L, R, \eta, p, q), L, R, \eta, p, p, q, q\right)+O\left(L^{\frac{4}{5}}\right) \\
& =1-\frac{4}{R} \sqrt{\frac{2}{C_{q}}} L^{\frac{1}{5}}+O\left(L^{\frac{2}{5}}\right) .
\end{aligned}
$$

Setting the coefficients of the terms $L^{\frac{1}{5}}$ equal, we obtain

$$
-2 \eta^{\frac{1}{2}} \frac{G_{\min }}{C_{p}}=-8 \sqrt{C_{q} C_{p}} R=-\frac{4}{R} \sqrt{\frac{2}{C_{q}}},
$$

whose solutions $C_{p}^{*}, C_{q}^{*}$ lead to the announced result for $p^{*}, q^{*}$, and $\bar{k}_{1,2}^{*}$.

TheOREM 3.12 (OO2 asymptotics). The parameters $p^{*}, q^{*}$ in (3.25) are for $L$ small solving the the min-max problem (3.23), and the associated convergence factor satisfies asymptotically

$$
\max _{k \geq k_{\min }}\left|\rho_{O O 2}\left(k, L, R, \eta, p^{*}, q^{*}\right)\right|=1-2^{\frac{12}{5}} \eta^{\frac{1}{10}} G_{\min }^{\frac{1}{5}} L^{\frac{1}{5}}+O\left(L^{\frac{2}{5}}\right) .
$$


Proof. Inserting the asymptotic values of $p^{*}, q^{*}$ given in Theorem 3.11 and $k=k_{\text {min }}$ into the convergence factor $\rho_{O O 2}(k, L, R, \eta, p, q)$ and expanding for $L$ small, we obtain $\rho_{\mathrm{OO} 2}\left(k_{\min }, L, R, \eta, p^{*}, q^{*}\right)=1-2^{\frac{12}{5}} \eta^{\frac{1}{10}} G_{\min }^{\frac{1}{5}} L^{\frac{1}{5}}+O\left(L^{\frac{2}{5}}\right)$. We thus need only show that $p^{*}, q^{*}$ given in Theorem 3.11 are solving the min-max problem (3.23) asymptotically. We denote $T_{1}(k, L, p, q):=\frac{-\sqrt{\eta} v_{k}(\sqrt{\eta}(R+L))-p-q k^{2}}{-\sqrt{\eta} v_{k}(\sqrt{\eta} R)+p+q k^{2}}$ and $T_{2}(k, L, p, q):=$ $\frac{\sqrt{\eta} w_{k}(\sqrt{\eta} R)-p-q k^{2}}{\sqrt{\eta} w_{k}(\sqrt{\eta}(R+L))+p+q k^{2}}$. Then $T_{1,2}\left(k_{\min }, L, p^{*}, q^{*}\right)<0, T_{1,2}\left(\bar{k}_{1}^{*}, L, p^{*}, q^{*}\right)>0, T_{1,2}\left(\bar{k}_{2}^{*}, L\right.$, $\left.p^{*}, q^{*}\right)<0$, since by Remark 3.7 we have $p^{*}+q^{*} k_{\min }^{2}>\max \left\{-v_{k_{\min }}(\sqrt{\eta}(R+\right.$ $\left.L)), w_{k_{m i n}}(\sqrt{\eta} R)\right\}, p^{*}+q^{*}\left(\bar{k}_{1}^{*}\right)^{2}<\min \left\{-v_{\bar{k}_{1}^{*}}(\sqrt{\eta}(R+L)), w_{\bar{k}_{1}^{*}}(\sqrt{\eta} R)\right\}$ and $p^{*}+q^{*}\left(\bar{k}_{2}^{*}\right)^{2}>$ $\max \left\{-v_{\bar{k}_{2}^{*}}(\sqrt{\eta}(R+L)), w_{\bar{k}_{2}^{*}}(\sqrt{\eta} R)\right\}$ for $L>0$ small. By the same technique applied in the proof of Theorem 3.9 we can show for $L$ small that $T_{1,2}\left(k, L, p^{*}, q^{*}\right)$ is negative and increasing in $k$ for $k<\underline{k}_{1}$, where $\underline{k}_{1}$ is defined in Lemma 3.10, and thus $\rho_{\text {OO } 2}\left(k_{\text {min }}, L, R, \eta, p^{*}, q^{*}\right)=T_{1}\left(k, \bar{L}, p^{*}, q^{*}\right) T_{2}\left(k, L, p^{*}, q^{*}\right) \rho_{c l a}(k, L, R, \eta)$ decreases in $k$ for $k<\underline{k}_{1}$, since $\rho_{\text {cla }}$ is always a decreasing function of $k$ for $k>0$. As a consequence, $k_{\text {min }}$ is a possible maximum point of $\rho_{O O 2}\left(k, L, R, \eta, p^{*}, q^{*}\right)$ for $L$ small enough, as well as the two interior maxima points $\bar{k}_{1,2}^{*}$ found earlier.

Let $p=C_{p} L^{-\alpha}, q=C_{q} L^{\beta}$ and $p^{*}=C_{p}^{*} L^{-\alpha^{*}}, q^{*}=C_{q}^{*} L^{\beta^{*}}$, where $\alpha^{*}=\frac{1}{5}, \beta^{*}=\frac{3}{5}$, as shown in Theorem 3.11. We now need to show that if $(p, q) \neq\left(p^{*}, q^{*}\right)$, then there exists a $k^{*}$ such that for $L>0$ small enough

$$
\rho_{O O 2}\left(k^{*}, L, R, \eta, p, q\right)>1-2 \eta^{\frac{1}{2}} \frac{G_{\min }}{C_{p}^{*}} L^{\frac{1}{5}}=1-8 \sqrt{C_{p}^{*} C_{q}^{*}} R L^{\frac{1}{5}}=1-\frac{4}{R} \sqrt{\frac{2}{C_{q}^{*}}} L^{\frac{1}{5}} .
$$

We first show that if $(\alpha, \beta) \neq\left(\alpha^{*}, \beta^{*}\right)$, then the asymptotic order of the resulting optimized Schwarz method will be enlarged. To this end, it is sufficient to treat the following cases:

(a) $\alpha>\alpha^{*}, \beta>-\alpha$. In this case at $k^{*}=k_{\text {min }}$ we have $\rho_{O O 2}\left(k^{*}, L, R, \eta, p, q\right)=$ $1-\frac{2 \sqrt{\eta} G_{\min }}{C_{p}} L^{\alpha}-\sqrt{\eta} G_{\min } L+o\left(L^{\min \{\alpha, 1\}}\right)$.

(b) $\alpha>\alpha^{*}, \beta=-\alpha$. In this case at $k^{*}=k_{\text {min }}$ we have $\rho_{O O 2}\left(k^{*}, L, R, \eta, p, q\right)=$ $1-\frac{2 \sqrt{\eta} G_{\min }}{C_{p}+C_{q} k_{\min }^{2}} L^{\alpha}-\sqrt{\eta} G_{\min } L+o\left(L^{\min \{\alpha, 1\}}\right)$.

(c) $\alpha>\alpha^{*}, \beta<-\alpha$. In this case at $k^{*}=k_{\text {min }}$ we have $\rho_{O O 2}\left(k^{*}, L, R, \eta, p, q\right)=$ $1-\frac{2 \sqrt{\eta} G_{\min }}{C_{q} k_{\min }^{2}} L^{-\beta}-\sqrt{\eta} G_{\min } L+o\left(L^{\min \{-\beta, 1\}}\right)$.

(d) $\alpha=\alpha^{*}, \beta>\beta^{*}$. Here we have to treat two cases: if $\beta \leq 1$, for $k^{*}=C_{k} L^{-\frac{\alpha+\beta}{2}}$ we have $\rho_{O O 2}\left(k^{*}, L, R, \eta, p, q\right)=\rho_{a p p}\left(k^{*}, L, R, \eta, p, p, q, q\right)+O\left(L^{\alpha+\beta}\right)=1-$ $\frac{4 R}{C_{k}}\left(C_{p}+C_{q} C_{k}^{2}\right) L^{\frac{\beta-\alpha}{2}}-\frac{2 C_{k}}{R} L^{\frac{2-\alpha-\beta}{2}}+o\left(L^{\frac{\beta-\alpha}{2}}\right)$. If $\beta>1$, we take $k^{*}=C_{k} L^{-\frac{3}{5}}$ and have $\rho_{O O 2}\left(k^{*}, L, R, \eta, p, q\right)=\rho_{a p p}\left(k^{*}, L, R, \eta, p, p, q, q\right)+O\left(L^{\frac{6}{5}}\right)=1-$ $\left(\frac{4 C_{p} R}{C_{k}}+\frac{2 C_{k}}{R}\right) L^{\frac{2}{5}}+o\left(L^{\frac{2}{5}}\right)$.

(e) $\alpha \leq \alpha^{*}, \beta<\beta^{*}$. In this case at $k^{*}=C_{k} L^{-\frac{\beta+1}{2}}$ we have $\rho_{O O 2}\left(k^{*}, L, R, \eta, p, q\right)=$ $\rho_{a p p}\left(k^{*}, L, R, \eta, p, p, q, q\right)+O\left(L^{\beta+1}\right)=1-2\left(\frac{2}{R C_{q} C_{k}}+\frac{C_{k}}{R}\right) L^{\frac{1-\beta}{2}}+o\left(L^{\frac{1-\beta}{2}}\right)$.

(f) $\alpha<\alpha^{*}, \beta>\beta^{*}$. In this case at $k^{*}=C_{k} L^{-\frac{2}{5}}$ we have $\rho_{O O 2}\left(k^{*}, L, R, \eta, p, q\right)=$ $\rho_{a p p}\left(k^{*}, L, R, \eta, p, p, q, q\right)+O\left(L^{\frac{4}{5}}\right)=1-\frac{4 R}{C_{k}}\left(C_{p} L^{\frac{2}{5}-\alpha}+C_{q} C_{k}^{2} L^{\beta-\frac{2}{5}}\right)-\frac{2 C_{k}}{R} L^{\frac{3}{5}}+$ $o\left(L^{\min \left\{\frac{2}{5}-\alpha, \beta-\frac{2}{5}, \frac{3}{5}\right\}}\right)$.

(g) $\alpha<\alpha^{*}, \beta=\beta^{*}$. In this case at $k^{*}=C_{k} L^{-\frac{\alpha+\beta}{2}}$ we have $\rho_{O O 2}\left(k^{*}, L, R, \eta, p, q\right)=$ $\rho_{a p p}\left(k^{*}, L, R, \eta, p, p, q, q\right)+O\left(L^{\alpha+\beta}\right)=1-\frac{4 R}{C_{k}}\left(C_{p}+C_{q} C_{k}^{2}\right) L^{\frac{\beta-\alpha}{2}}-\frac{2 C_{k}}{R} L^{\frac{2-\alpha-\beta}{2}}+$ $o\left(L^{\frac{\beta-\alpha}{2}}\right)$.

We therefore see that in each case above, at the given $k^{*}$ the convergence factor 
$\rho_{\mathrm{OO} 2}\left(k^{*}, L, R, \eta, p, q\right)$ behaves asymptotically like $1-C L^{\delta}$ with $\delta>\frac{1}{5}$.

It remains thus to consider the case $\alpha=\alpha^{*}$ and $\beta=\beta^{*}$ but $\left(C_{p}, C_{q}\right) \neq\left(C_{p}^{*}, C_{q}^{*}\right)$. From (3.27) we see $\rho_{\mathrm{OO} 2}\left(k_{\text {min }}, L, R, \eta, p, q\right)>\rho_{\mathrm{OO} 2}\left(k_{\text {min }}, L, R, \eta, p^{*}, q^{*}\right)$ asymptotically if $C_{p}>C_{p}^{*}$. From (3.29) we see $\rho_{O O 2}\left(\bar{k}_{2}, L, R, \eta, p, q\right)>\rho_{O O 2}\left(\bar{k}_{2}^{*}, L, R, \eta, p^{*}, q^{*}\right)$ if $C_{q}>C_{q}^{*}$, and from (3.28) we conclude that $\rho_{\mathrm{OO} 2}\left(\bar{k}_{1}, L, R, \eta, p, q\right)>\rho_{\mathrm{OO} 2}\left(\bar{k}_{2}^{*}, L, R\right.$, $\left.\eta, p^{*}, q^{*}\right)$ if $C_{p}<C_{p}^{*}$ or $C_{q}<C_{q}^{*}$, which concludes the proof.

3.4. A two-sided optimized Robin transmission condition. We now consider a two-sided optimized Robin transmission condition, $p_{1}>0, p_{2}>0, q_{1}=q_{2}=0$, which can better take into account the difference in the Robin transmission conditions we have seen due to curvature in subsection 3.1. We obtain for this choice from (3.5) the convergence factor

$$
\begin{aligned}
\rho_{O 2 s}\left(k, L, R, \eta, p_{1}, p_{2}\right)= & \frac{-\sqrt{\eta} K_{k}^{\prime}(\sqrt{\eta}(R+L))-p_{1} K_{k}(\sqrt{\eta}(R+L))}{\sqrt{\eta} I_{k}^{\prime}(\sqrt{\eta}(R+L))+p_{1} I_{k}(\sqrt{\eta}(R+L))} \\
& \frac{\sqrt{\eta} I_{k}^{\prime}(\sqrt{\eta} R)-p_{2} I_{k}(\sqrt{\eta} R)}{-\sqrt{\eta} K_{k}^{\prime}(\sqrt{\eta} R)+p_{2} K_{k}(\sqrt{\eta} R)} .
\end{aligned}
$$

To determine optimized parameters $p_{1}^{*}$ and $p_{2}^{*}$ for the associated Schwarz method, we have to solve the min-max problem

$$
\min _{p_{1}, p_{2}>0}\left(\max _{k \geq k_{\min }}\left|\rho_{O 2 s}\left(k, L, R, \eta, p_{1}, p_{2}\right)\right|\right) .
$$

Theorem 3.13 (optimized two-sided Robin parameters). The parameters

$$
p_{1}^{*}=2^{-\frac{8}{5}} \eta^{\frac{2}{5}} G_{\min }^{\frac{4}{5}} L^{-\frac{1}{5}}, \quad p_{2}^{*}=2^{-\frac{4}{5}} \eta^{\frac{1}{5}} G_{\min }^{\frac{2}{5}} L^{-\frac{3}{5}}
$$

are for $L$ small solutions of the equioscillation equations

$$
\begin{aligned}
\rho_{O 2 s}\left(k_{m i n}, L, R, \eta, p_{1}^{*}, p_{2}^{*}\right) & =-\rho_{O 2 s}\left(\hat{k}_{1}^{*}, L, R, \eta, p_{1}^{*}, p_{2}^{*}\right) \\
& =\rho_{O 2 s}\left(\hat{k}_{2}^{*}, L, R, \eta, p_{1}^{*}, p_{2}^{*}\right),
\end{aligned}
$$

where $\hat{k}_{1,2}^{*}=\hat{k}_{1,2}^{*}\left(L, R, \eta, p_{1}, p_{2}\right)$, at which $\rho_{O 2 s}\left(k, L, R, \eta, p_{1}^{*}, p_{2}^{*}, 0,0\right)$ attains its interior minimum and maximum asymptotically, with $\hat{k}_{1}^{*} \sim 2^{-\frac{6}{5}} \eta^{\frac{3}{10}} G_{\min }^{\frac{3}{5}} R L^{-\frac{2}{5}}$ and $\hat{k}_{2}^{*} \sim 2^{-\frac{2}{5}} \eta^{\frac{1}{10}} G_{\min }^{\frac{1}{5}} R L^{-\frac{4}{5}}$.

Proof. We first show that the convergence factor $\rho_{a p p}\left(k, L, R, \eta, p_{1}^{*}, p_{2}^{*}, 0,0\right)$ attains its interior minimum and maximum asymptotically at $\hat{k}_{1}=2^{-\frac{6}{5}} \eta^{\frac{3}{10}} G_{\min }^{\frac{3}{5}} R L^{-\frac{2}{5}}$ and $\hat{k}_{2}=2^{-\frac{2}{5}} \eta^{\frac{1}{10}} G_{\min }^{\frac{1}{5}} R L^{-\frac{4}{5}}$, and then $\rho_{O 2 s}\left(k, L, R, \eta, p_{1}^{*}, p_{2}^{*}\right)$ does as well, because of Theorem 3.3. Solving $\partial_{k} \rho_{a p p}\left(k, L, R, \eta, p_{1}, p_{2}, 0,0\right)=0$, we get the positive solutions

$$
\hat{k}_{1,2}\left(L, R, \eta, p_{1}, p_{2}\right)=\frac{\sqrt{2} R}{2} \sqrt{\frac{L p_{1}^{2}+L p_{2}^{2}-2 L \eta+p_{2}+p_{1} \mp \sqrt{\Phi}}{L}},
$$

where $\Phi=p_{2}^{2}+p_{1}^{2}+2 p_{1} p_{2}-2 L^{2} p_{1}^{2} p_{2}^{2}-2 L p_{1}^{2} p_{2}-2 L p_{2}^{2} p_{1}+L^{2} p_{1}^{4}+2 L p_{1}^{3}+L^{2} p_{2}^{4}+2 L p_{2}^{3}$. Inserting the ansatz $p_{1}=C_{1} L^{-\frac{1}{5}}$ and $p_{2}=C_{2} L^{-\frac{3}{5}}$ and expanding for $L$ small, we get $\hat{k}_{1}=R \sqrt{C_{1} C_{2}} L^{-\frac{2}{5}}+O(1)$ and $\hat{k}_{2}=R \sqrt{C_{2}} L^{-\frac{4}{5}}+O\left(L^{-\frac{2}{5}}\right)$. Using the same ansatz in $\rho_{O 2 s}\left(k_{\text {min }}, L, R, \eta, p_{1}, p_{2}\right)$ and expanding for $L$ small gives

$$
\rho_{O 2 s}\left(k_{m i n}, L, R, \eta, p_{1}, p_{2}\right)=1-\frac{\eta^{\frac{1}{2}} G_{m i n}}{C_{1}} L^{\frac{1}{5}}+O\left(L^{\frac{2}{5}}\right) .
$$


Inserting this ansatz and the expressions for $\hat{k}_{1,2}$ into $-\rho_{\text {app }}\left(\hat{k}_{1}, L, R, \eta, p_{1}, p_{2}, 0,0\right)$ and $\rho_{\text {app }}\left(\hat{k}_{2}, L, R, \eta, p_{1}, p_{2}, 0,0\right)$ and expanding for $L$ small, we obtain in addition

$$
\begin{aligned}
-\rho_{a p p}\left(\hat{k}_{1}, L, R, \eta, p_{1}, p_{2}, 0,0\right) & =1-4 \sqrt{\frac{C_{1}}{C_{2}}} L^{\frac{1}{5}}+O\left(L^{\frac{2}{5}}\right), \\
\rho_{\text {app }}\left(\hat{k}_{2}, L, R, \eta, p_{1}, p_{2}, 0,0\right) & =1-4 \sqrt{C_{2}} L^{\frac{1}{5}}+O\left(L^{\frac{2}{5}}\right) .
\end{aligned}
$$

Therefore, by Theorem 3.3 we have

$$
\begin{aligned}
-\rho_{O 2 s}\left(\hat{k}_{1}, L, R, \eta, p_{1}, p_{2}\right) & =-\rho_{\text {app }}\left(\hat{k}_{1}, L, R, \eta, p_{1}, p_{2}, 0,0\right)+O\left(\frac{1}{\hat{k}_{1}^{2}}+\frac{L}{\hat{k}_{1}}+L^{2}\right) \\
& =1-4 \sqrt{\frac{C_{1}}{C_{2}}} L^{\frac{1}{5}}+O\left(L^{\frac{2}{5}}\right), \\
\rho_{O 2 s}\left(\hat{k}_{2}, L, R, \eta, p_{1}, p_{2}\right) & =\rho_{\text {app }}\left(\hat{k}_{2}, L, R, \eta, p_{1}, p_{2}, 0,0\right)+O\left(\frac{1}{\hat{k}_{2}^{2}}+\frac{L}{\hat{k}_{2}}+L^{2}\right) \\
& =1-4 \sqrt{C_{2}} L^{\frac{1}{5}}+O\left(L^{\frac{2}{5}}\right) .
\end{aligned}
$$

Setting the coefficients of the terms $L^{\frac{1}{5}}$ in (3.37), (3.38), and (3.39) equal, we obtain

$$
\frac{\eta^{\frac{1}{2}} G_{\min }}{C_{1}}=4 \sqrt{\frac{C_{1}}{C_{2}}}=4 \sqrt{C_{2}} .
$$

Denoting by $C_{1}^{*}=2^{-\frac{8}{5}} \eta^{\frac{2}{5}} G_{\text {min }}^{\frac{4}{5}}$ and $C_{2}^{*}=2^{-\frac{4}{5}} \eta^{\frac{1}{5}} G_{\text {min }}^{\frac{2}{5}}$ the solutions of (3.40), the result (3.35) follows.

Remark 3.14. From the proof of the above theorem, we see that if we swap the parameters $p_{1}^{*}$ and $p_{2}^{*}$, i.e., taking $p_{1}^{*}=2^{-\frac{4}{5}} \eta^{\frac{1}{5}} G_{\text {min }}^{\frac{2}{5}} L^{-\frac{3}{5}}$ and $p_{2}^{*}=2^{-\frac{8}{5}} \eta^{\frac{2}{5}} G_{\text {min }}^{\frac{4}{5}} L^{-\frac{1}{5}}$, the problem (3.36) is solved asymptotically as well for $L$ small enough.

THEOREM 3.15 (2s asymptotics). The parameter values of $p_{1}^{*}, p_{2}^{*}$ in (3.35) solve the min-max problem (3.34) asymptotically, and the associated convergence factor satisfies for $L$ small

$$
\max _{k \geq k_{\min }}\left|\rho_{O 2 s}\left(k, L, R, \eta, p_{1}^{*}, p_{2}^{*}\right)\right|=1-2^{\frac{8}{5}} \eta^{\frac{1}{10}} G_{\min }^{\frac{1}{5}} L^{\frac{1}{5}}+O\left(L^{\frac{2}{5}}\right) .
$$

Proof. Let $T_{1}\left(k, L, p_{1}, p_{2}\right):=\left(-\sqrt{\eta} v_{k}(\sqrt{\eta}(R+L))-p_{1}\right) /\left(-\sqrt{\eta} v_{k}(\sqrt{\eta} R)+p_{2}\right)$ and $T_{2}\left(k, L, p_{1}, p_{2}\right):=\left(\sqrt{\eta} w_{k}(\sqrt{\eta} R)-p_{2}\right) /\left(\sqrt{\eta} w_{k}(\sqrt{\eta}(R+L))+p_{1}\right)$, which implies $\rho_{O 2 s}\left(k, L, R, \eta, p_{1}, p_{2}\right)=T_{1}\left(k, L, p_{1}, p_{2}\right) T_{2}\left(k, L, p_{1}, p_{2}\right) \rho_{c l a}(k, L, R, \eta)$. Let $\underline{k}_{1,2}$ be such that $-\sqrt{\eta} \underline{\underline{k}}_{1}(\sqrt{\eta}(R+L))=p_{1}^{*}$ and $\sqrt{\eta} w_{\underline{k}_{2}}(\sqrt{\eta} R)=p_{2}^{*}$. From Lemma 3.6 we know that $\underline{k}_{1} \sim R p_{1}^{*}=2^{-\frac{8}{5}} R \eta^{\frac{2}{5}} G_{\min }^{\frac{4}{5}} L^{-\frac{1}{5}}, \underline{k}_{2} \sim R p_{2}^{*}=2^{-\frac{4}{5}} R \eta^{\frac{1}{5}} G_{\text {min }}^{\frac{2}{5}} L^{-\frac{3}{5}}$, and thus $\underline{k}_{1}<$ $\underline{k}_{2}$ for $L$ small enough. Now if $k<\underline{k}_{1}$, by the same argument used in the proof of Theorem 3.9 we know that $T_{1}\left(k, L, p_{1}^{*}, p_{2}^{*}\right)$ and $T_{2}\left(k, L, p_{1}^{*}, p_{2}^{*}\right)$ are negative increasing functions of $k$ for $L$ small because of the monotonicity of $v_{k}(x)$ and $w_{k}(x)$ shown in Theorem A.3. Since $\rho_{\text {cla }}(k, L, R, \eta)$ decreases in $k$ for $L$ small, the convergence factor $\rho_{O 2 s}\left(k, L, R, \eta, p_{1}^{*}, p_{2}^{*}\right)$, as a product of these three terms, decreases in $k$ for $k_{\min } \leq k \leq \underline{k}_{1}$ and $L$ small, and hence $k=k_{\text {min }}$ is a possible maximum point of $\rho_{O 2 s}\left(k, L, R, \eta, p_{1}^{*}, p_{2}^{*}\right)$ for $L$ small.

When $k>\underline{k}_{1}$, we have $\rho_{O 2 s}\left(k, L, R, \eta, p_{1}^{*}, p_{2}^{*}\right)=\rho_{a p p}\left(k, L, R, \eta, p_{1}^{*}, p_{2}^{*}, 0,0\right)+$ $O\left(L^{-\frac{2}{5}}\right)$. Since $\rho_{\text {app }}\left(k, L, R, \eta, p_{1}^{*}, p_{2}^{*}, 0,0\right)$ attains its unique interior minimum asymptotically at $\bar{k}_{1}$ and attains its unique interior maximum asymptotically at $\bar{k}_{2}$, we know from Theorem 3.3 that $\rho_{O 2 s}\left(k, L, R, \eta, p_{1}^{*}, p_{2}^{*}\right)$ also attains its interior minimum and maximum asymptotically at $\bar{k}_{1}, \bar{k}_{2}$. 
We now show that $p_{1}^{*}$ and $p_{2}^{*}$ given in Theorem 3.13 solve the min-max problem (3.34) asymptotically. We make the ansatz $p_{1}=C_{1} L^{-\alpha}, p_{2}=C_{2} L^{-\beta}$ and set $p_{1}^{*}=C_{1}^{*} L^{-\alpha^{*}}, p_{2}^{*}=C_{2}^{*} L^{-\beta^{*}}$ with $\alpha^{*}=\frac{1}{5}, \beta^{*}=\frac{3}{5}$. With this we obtain $\rho_{O 2 s}\left(k_{m i n}, L, R, \eta, p_{1}^{*}, p_{2}^{*}\right)=1-2^{\frac{8}{5}} \eta^{\frac{1}{10}} G_{m i n}^{\frac{1}{5}} L^{\frac{1}{5}}+O\left(L^{\frac{2}{5}}\right)$, and we have to show that this is the optimal asymptotic convergence factor. We first prove that if $(\alpha, \beta) \neq\left(\alpha^{*}, \beta^{*}\right)$, there exists a frequency $k^{*}$ such that at this frequency the convergence factor has an asymptotic order bigger than $\frac{1}{5}$. We show this by examining in detail the following cases, where due to Remark 3.14, we consider only the case $\beta \geq \alpha$; when $\beta<\alpha$, we could consider the case where $p_{1}, p_{2}$ are swapped.

(a) $\alpha>\alpha^{*}, \beta>\alpha$. In this case at $k^{*}=k_{\min }$ we have $\rho_{O 2 s}\left(k^{*}, L, R, \eta, p_{1}, p_{2}\right)=$ $1-\sqrt{\eta} \frac{G_{\min }}{C_{1}} L^{\alpha}-\sqrt{\eta} G_{\min } L+o\left(L^{\min \{\alpha, 1\}}\right)$.

(b) $\beta=\alpha>\alpha^{*}$. In this case at $k^{*}=k_{\min }$ we have $\rho_{O 2 s}\left(k^{*}, L, R, \eta, p_{1}, p_{2}\right)=$ $1-\sqrt{\eta} G_{\min }\left(\frac{1}{C_{1}}+\frac{1}{C_{2}}\right) L^{\alpha}-\sqrt{\eta} G_{\min } L+o\left(L^{\min \{\alpha, 1\}}\right)$.

(c) $\alpha<\alpha^{*}, \beta>\beta^{*}$. In this case at $k^{*}=C_{k} L^{-\frac{2}{5}}$ we have $\rho_{O 2 s}\left(k^{*}, L, R, \eta, p_{1}, p_{2}\right)=$ $-1+2 \frac{C_{1} R}{C_{k}} L^{\frac{2}{5}-\alpha}+2 \frac{C_{k}}{R C_{2}} L^{\beta-\frac{2}{5}}+2 \frac{C_{k}}{R} L^{\frac{3}{5}}+o\left(L^{\min \left\{\frac{2}{5}-\alpha, \beta-\frac{2}{5}, \frac{3}{5}\right\}}\right)$.

(d) $\alpha \leq \alpha^{*}, \alpha \leq \beta<\beta^{*}$. In this case at $k^{*}=C_{k} L^{-\frac{\beta+1}{2}}$ we obtain that $\rho_{O 2 s}\left(k^{*}, L, R, \eta, p_{1}, p_{2}\right)=1-2 \frac{R C_{1}}{C_{k}} L^{\frac{\beta+1-2 \alpha}{2}}-2\left(\frac{C_{2} R}{C_{k}}+\frac{C_{k}}{R}\right) L^{\frac{1-\beta}{2}}+o\left(L^{\frac{1-\beta}{2}}\right)$.

(e) $\alpha=\alpha^{*}, \beta>\beta^{*}$. If $\beta \leq 1$, we have $\rho_{O 2 s}\left(C_{k} L^{-\frac{\alpha+\beta}{2}}, L, R, \eta, p_{1}, p_{2}\right)=-1+$ $2\left(\frac{C_{1} R}{C_{k}}+\frac{C_{k}}{R C_{2}}\right) L^{\frac{\beta-\alpha}{2}}+2 \frac{C_{k}}{R} L^{\frac{2-\alpha-\beta}{2}}+o\left(L^{\frac{\beta-\alpha}{2}}\right)$, and if $\beta>1$, we obtain that $\rho_{O 2 s}\left(C_{k} L^{-\frac{3}{5}}, L, R, \eta, p_{1}, p_{2}\right)=-1+2\left(\frac{C_{1} R}{C_{k}}+\frac{C_{k}}{R}\right) L^{\frac{2}{5}}+o\left(L^{\frac{2}{5}}\right)$.

(f) $\beta=\beta^{*}, \alpha<\alpha^{*}$. We have that $\rho_{O 2 s}\left(C_{k} L^{-\frac{\alpha+\beta}{2}}, L, R, \eta, p_{1}, p_{2}\right)=-1+2\left(\frac{C_{1} R}{C_{k}}+\right.$ $\left.\frac{C_{k}}{R C_{2}}\right) L^{\frac{\beta-\alpha}{2}}+o\left(L^{\frac{\beta-\alpha}{2}}\right)$.

In each of the cases above, we see that at $k^{*}$ the convergence factor $\left|\rho_{O 2 s}\left(k^{*}, L, R, \eta, p, q\right)\right|$ behaves asymptotically like $1-C L^{\delta}$ with $\delta>\frac{1}{5}$.

We finally consider the case $(\alpha, \beta)=\left(\alpha^{*}, \beta^{*}\right)$ but $\left(C_{1}, C_{2}\right) \neq\left(C_{1}^{*}, C_{2}^{*}\right)$. If $C_{1}>$ $C_{1}^{*}$, we get from (3.37) that $\rho_{O 2 s}\left(k_{m i n}, L, R, \eta, p_{1}, p_{2}\right)>\rho_{O 2 s}\left(k_{m i n}, L, R, \eta, p_{1}^{*}, p_{2}^{*}\right)$, and if $C_{2}<C_{2}^{*}$, we get from (3.39) that $\rho_{O 2 s}\left(\hat{k}_{2}, L, R, \eta, p_{1}, p_{2}\right)>\rho_{O 2 s}\left(\hat{k}_{2}^{*}, L, R, \eta, p_{1}^{*}, p_{2}^{*}\right)$, both for $L>0$ small enough. The last case we need to consider is $C_{1}<C_{1}^{*}$ and $C_{2}>C_{2}^{*}$; then $\frac{C_{1}}{C_{2}}<\frac{C_{1}^{*}}{C_{2}^{*}}$, and thus from (3.38) we have $\left|\rho_{O 2 s}\left(\hat{k}_{1}, L, R, \eta, p_{1}, p_{2}\right)\right|>$ $\left|\rho_{O 2 s}\left(\hat{k}_{1}^{*}, L, R, \eta, p_{1}^{*}, p_{2}^{*}\right)\right|$ for $L>0$ small enough, which concludes the proof.

4. Numerical experiments. We show two sets of experiments for the model problem (1.1) - one on a circular domain, a situation close to our analysis, and one on a rectangular domain with a wavy interface. We use a linear finite element method with uniform mesh in each subdomain and FreeFem ++ for the simulations. We directly numerically study the error equations, $f=0$, and use a random initial guess so that all the frequency components are present; see [18, section 5] for the importance of this.

4.1. Circular domain. The domain here is the unit disk $\Omega=[0, M) \times[0,2 \pi)$, decomposed into $\Omega=\Omega_{1} \cup \Omega_{2}$ with $\Omega_{1}=[0, R+L] \times[0,2 \pi)$ and $\Omega_{2}=[R, M] \times[0,2 \pi)$, where $R=0.5$ and $L>0$ is the overlap. We choose $\eta=2$ and numerically investigate the two cases of a relatively large domain and a relatively small domain, with the overlap $L=h$, the finite element mesh size. This is the typical case in practical applications, where the overlapping optimized Schwarz methods need almost the same amount of computation as the nonoverlapping ones but converge faster; see [24]. 


\begin{tabular}{|l|c|c|c|c|c|}
\hline $\mathrm{h}$ & $\frac{1}{16}$ & $\frac{1}{32}$ & $\frac{1}{64}$ & $\frac{1}{128}$ & $\frac{1}{256}$ \\
\hline \hline Classical & 56 & 113 & 227 & 455 & 911 \\
\hline T0 & 8 & 11 & 16 & 24 & 35 \\
\hline AT0 & 8 & 11 & 16 & 23 & 35 \\
\hline AT2 & 8 & 8 & 9 & 11 & 15 \\
\hline OO0 & 5 & 7 & 10 & 13 & 17 \\
\hline OO2 & 6 & 6 & 6 & 7 & 8 \\
\hline O2s & 9 & 8 & 7 & 9 & 10 \\
\hline
\end{tabular}

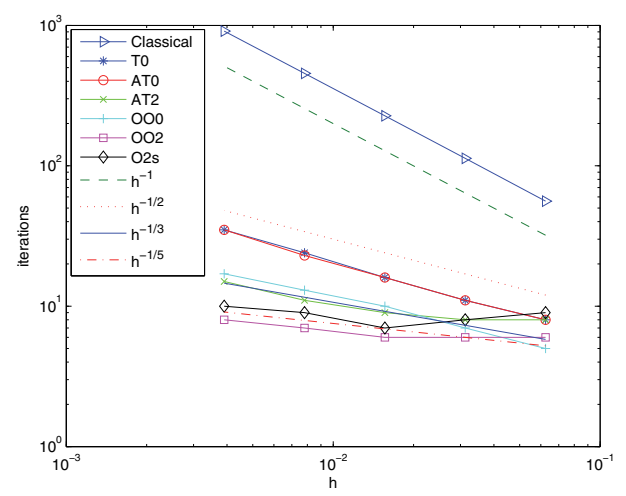

FIG. 4. Number of iterations required by the Schwarz methods for a relatively large domain.
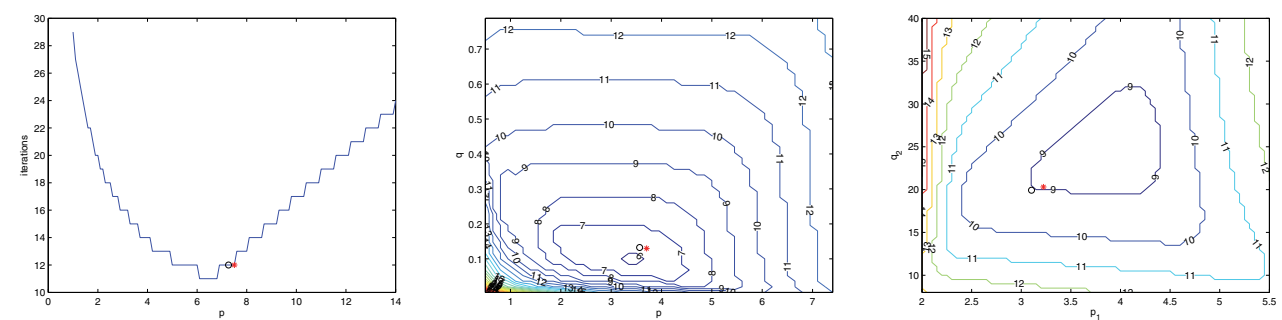

FIG. 5. Optimized parameter (*) found by the analytical optimization compared to the appropriately scaled optimized parameter for a straight line interface (o) reported in [17], and to the performance of other values of the parameters: On the left for the Robin case, in the middle for the second order case, and on the right for two-sided Robin.

4.1.1. Relatively large domain. The domain size is $M=1$, and the subdomain size is $R=0.5$. We show in Figure 4 the iteration numbers required by the various Schwarz methods as a function of the mesh size $h$. These results illustrate well our asymptotic analysis. Note that the AT2 has not yet reached its asymptotic regime.

We next investigate how well the continuous analysis predicts the optimal parameters to be used in the numerical setting. To this end, we vary the parameter $p$ in the Robin transmission conditions for a fixed problem of mesh size $h=1 / 128$ and count for each value of $p$ the number of iterations to reach a residual of $1 e-6$, and similarly for the other transmission conditions. The results are shown in Figure 5.

These results show that the analysis predicts the optimal parameter very well.

In each plot, we also compare the optimized parameters obtained from the circular domain decomposition analysis to those obtained from the straight line interface analysis [17], which are indicated by "o", where we used the interface curvature in a heuristic fashion we learned from our analysis to obtain a good method: we estimate the lowest frequency involved by $1 / R$. We see that in this case there is no significant difference between the optimized parameters obtained by these two approaches.

4.1.2. Relatively small domain. We now choose a domain size $M=0.1$, $R=0.05$. The number of iterations required by the Schwarz methods is shown in Figure 6 . 


\begin{tabular}{|l|c|c|c|c|c|}
\hline $\mathrm{h}$ & $\frac{1}{160}$ & $\frac{1}{320}$ & $\frac{1}{640}$ & $\frac{1}{1280}$ & $\frac{1}{2560}$ \\
\hline \hline Classical & 70 & 144 & 290 & 579 & 1160 \\
\hline T0 & 18 & 23 & 32 & 46 & 66 \\
\hline AT0 & 41 & 67 & 81 & 86 & 105 \\
\hline AT2 & 49 & 67 & 79 & 86 & 97 \\
\hline OO0 & 5 & 7 & 10 & 14 & 17 \\
\hline OO2 & 8 & 7 & 6 & 7 & 8 \\
\hline O2s & 14 & 11 & 9 & 9 & 12 \\
\hline
\end{tabular}

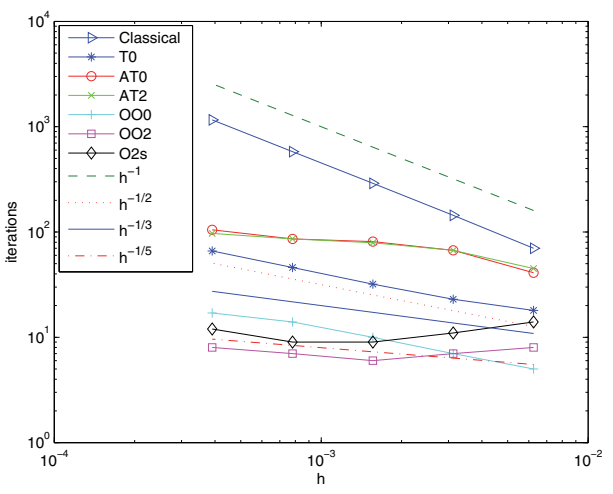

FIG. 6. Number of iterations required by the Schwarz methods for a relatively small domain.
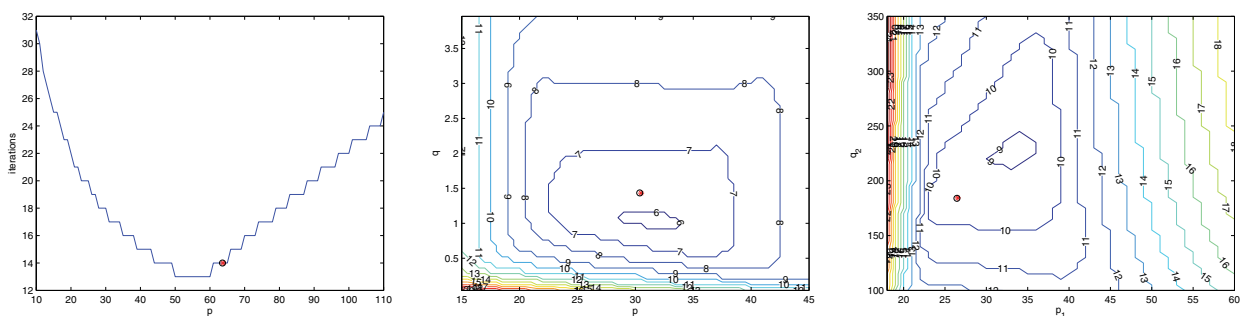

FIG. 7. Optimized parameter (*) found by the analytical optimization compared to the appropriately scaled optimized parameter for a straight line interface (o) reported in [17], and to the performance of other values of the parameters: On the left for the Robin case, in the middle for the second order case, and on the right for two-sided Robin, for the relatively small domain.

We see that the approximate Taylor conditions have not yet reached their asymptotic regime.

We next investigate, as we did for the relatively large domain, how well the continuous analysis predicts the optimal parameters to be used in the numerical setting for a fixed mesh size $h=1 / 1280$. The results are shown in Figure 7 and show that the analysis predicts the optimal parameter very well also. Again, we compare them to the appropriately scaled optimized parameters obtained from the straight interface analysis [17] and see that the good scaling revealed leads to a very similar performance.

4.2. Square domain. We consider now the model problem (1.1) on the square domain $\Omega=[0,1] \times[0,1]$, decomposed into $\Omega_{1} \cup \Omega_{2}$ as shown in Figure 8 .

The interfaces between subdomains $\Omega_{1}$ and $\Omega_{2}$ are described by the Sine functions $a+A \sin (2 \pi \omega y)$ and $b+A \sin (2 \pi \omega y)$, where we chose $a=0.5$ and $b=a+L$ and for the amplitude $A=0.1$. This model problem allows us to test our optimized transmission conditions in a situation where our analysis does not apply, but the results we obtained can nevertheless be used: we simply replace the radius of the circular interface in our optimized formulas for the parameters by the local radius of curvature along the interface. For various degrees of oscillation $\omega=1,5,10$, the numbers of iterations needed by the different Schwarz methods are listed in Table 1 with fixed overlap $L=1 / 32$ and in Table 2 for $L=h$. 


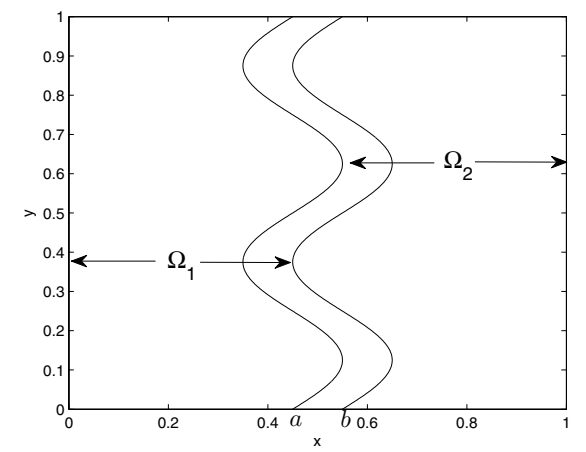

FIG. 8. Domain decomposition for Example 2.

TABLE 1

Number of iterations required by the Schwarz algorithms with different transmission conditions for the square domain case with fixed overlap $L=1 / 32$.

\begin{tabular}{|l|ccc|cccc|cccc|ccc|ccc|}
\hline$h$ & \multicolumn{3}{|c|}{$1 / 32$} & \multicolumn{3}{|c|}{$1 / 64$} & \multicolumn{4}{|c|}{$1 / 128$} & \multicolumn{3}{|c|}{$1 / 256$} & \multicolumn{3}{|c|}{$1 / 512$} \\
\hline$\omega$ & 1 & 5 & 10 & 1 & 5 & 10 & 1 & 5 & 10 & 1 & 5 & 10 & 1 & 5 & 10 \\
\hline \hline Classical & 59 & 228 & 602 & 59 & 220 & 634 & 59 & 220 & 665 & 59 & 220 & 649 & 59 & 220 & 651 \\
\hline T0 & 12 & 19 & $/$ & 13 & 24 & 383 & 12 & 28 & $>3000$ & 13 & 26 & $/$ & 13 & 26 & $/$ \\
\hline AT0 & 14 & 19 & 19 & 13 & 22 & 35 & 13 & 27 & 51 & 13 & 26 & 58 & 13 & 27 & 58 \\
\hline AT2 & 12 & 18 & 40 & 11 & 19 & 25 & 11 & 18 & 22 & 11 & 18 & 19 & 12 & 19 & 23 \\
\hline OO0 & 8 & 14 & 24 & 8 & 14 & 22 & 8 & 15 & 23 & 8 & 14 & 25 & 8 & 14 & 25 \\
\hline OO2 & 4 & 11 & 20 & 4 & 9 & 18 & 4 & 10 & 17 & 4 & 10 & 18 & 4 & 11 & 18 \\
\hline O2s & 8 & 22 & $/$ & 7 & 23 & $/$ & 8 & 26 & $/$ & 8 & 23 & $/$ & 7 & 25 & $/$ \\
\hline
\end{tabular}

We see first that with the overlap fixed, the number of iterations becomes constant as the mesh is refined, as predicted by our theorems, and that the optimized transmission conditions work better than those obtained from microlocal analysis and much better than the classical Schwarz algorithm. We observe as well that when the interface curvature is varying rapidly, the Schwarz algorithms require more iterations for each transmission condition. The extreme cases are the Schwarz algorithm with Taylor transmission condition of order 0 and optimized two-sided transmission condition, when the interface curvature varies very rapidly, i.e., $\omega=10$, and the methods fail to converge. This, however, only further confirms our finding that already in the circular domain decomposition not all positive parameters lead to convergent Schwarz algorithms. As far as the asymptotic performance is concerned, we see that the Schwarz method with optimized Robin and with optimized second order parameters works well for each case, even when the interface curvature varies very rapidly, i.e., $\omega=10$, and follows the asymptotic prediction from the circular decomposition when the mesh size becomes small. The Schwarz algorithm with optimized two-sided Robin transmission condition gives good results only for slow variation $\omega=1$, while when the variation is rapid it fails to converge. This is not surprising, however, because we have observed that in that case the parameters need to be swapped depending on the curvature [25], and this is difficult for a general interface.

5. Conclusion. We analyzed the optimized Schwarz methods applied to a model problem with circular domain decomposition. We showed that the curvature enters the optimized parameters and presented a new approach for analyzing best approxi- 
TABLE 2

Number of iterations required by the Schwarz algorithms with different transmission conditions for the square domain case with overlap $L=h$.

\begin{tabular}{|l|ccc|cccc|ccc|ccc|ccc|}
\hline$h$ & \multicolumn{3}{|c|}{$1 / 32$} & \multicolumn{3}{|c|}{$1 / 64$} & \multicolumn{3}{|c|}{$1 / 128$} & \multicolumn{3}{|c|}{$1 / 256$} & \multicolumn{3}{|c|}{$1 / 512$} \\
\hline$\omega$ & 1 & 5 & 10 & 1 & 5 & 10 & 1 & 5 & 10 & 1 & 5 & 10 & 1 & 5 & 10 \\
\hline \hline Classical & 59 & 231 & 605 & 110 & 423 & 1104 & 206 & 778 & 2130 & 383 & 1436 & $>3000$ & 713 & 2625 & $>3000$ \\
\hline T0 & 12 & 18 & $/$ & 20 & 26 & $/$ & 31 & 35 & $/$ & 46 & 46 & $/$ & 67 & 61 & $/$ \\
\hline AT0 & 14 & 16 & 23 & 21 & 23 & 34 & 30 & 35 & 52 & 44 & 49 & 66 & 64 & 70 & 80 \\
\hline AT2 & 12 & 20 & 45 & 15 & 39 & 63 & 24 & 59 & 81 & 38 & 96 & 103 & 54 & 163 & 253 \\
\hline OO0 & 9 & 16 & 26 & 12 & 21 & 37 & 15 & 30 & 48 & 20 & 40 & 69 & 27 & 50 & 92 \\
\hline OO2 & 4 & 11 & 20 & 5 & 13 & 18 & 6 & 15 & 24 & 6 & 18 & 30 & 7 & 25 & 39 \\
\hline O2s & 8 & 21 & $/$ & 10 & 40 & $/$ & 12 & 85 & $/$ & 14 & 2997 & $/$ & 17 & $/$ & $/$ \\
\hline
\end{tabular}

mation problems based on asymptotically accurate approximate convergence factors, obtained using Turán-type inequalities. Our analysis revealed that in the circular decomposition case, the optimized Schwarz methods are not necessarily convergent for all admissible parameters, which is an important difference from the straight interface case. We also derived closed form asymptotic formulas for transmission conditions of Robin and second order type. Even though the analysis is technical and difficult, the use of the optimized conditions is not; one can simply use the asymptotic formulas we obtained. Numerically we showed that they perform well, both on a model problem close to the analysis and in a more general case. We also learned from our analysis that one can rescale the optimized parameters obtained from the straight interface analysis to obtain a very good parameter choice taking into account the curvature of the interface. In the last experiment, we observed that the two-sided Robin condition performs less well than in the model problem setting. We think that this is because the parameters should be swapped depending on the interface curvature [25], which deserves further investigation. In the last domain decomposition conference DD22, we heard a presentation by Vergara about optimized Schwarz methods for decompositions with cylindrical interfaces [26], where the authors propose to optimize the performance for a particular frequency, but similar techniques to the ones we presented in this paper could be applied.

\section{Appendix. Monotonicity of $\frac{I_{k}^{\prime}(x)}{I_{k}(x)}$ and $\frac{K_{k}^{\prime}(x)}{K_{k}(x)}$ in the order $k$.}

Letting $w_{k}(x):=\frac{I_{k}^{\prime}(x)}{I_{k}(x)}$, a direct calculation shows that $w_{k}(x)$ satisfies the differential equation

$$
w_{k}^{\prime}+w_{k}^{2}+\frac{1}{x} w_{k}-\left(1+\frac{k^{2}}{x^{2}}\right)=0
$$

Rewriting this equation as

$$
w_{k}^{\prime}=-w_{k}^{2}-\frac{1}{x} w_{k}+1+\frac{k^{2}}{x^{2}}=: f_{k}\left(x, w_{k}\right),
$$

we note that for $k_{2}>k_{1}$ we have $f_{k_{2}}(x, w)>f_{k_{1}}(x, w)$.

Lemma A.1. There exists an $x_{0}, 0<x_{0} \ll 1$, such that $w_{k_{2}}\left(x_{0}\right)>w_{k_{1}}\left(x_{0}\right)$ for all $k_{2}>k_{1} \geq 0$.

Proof. For $k_{2}>k_{1} \geq 0$, we choose $x_{0}>0$ such that $\sqrt{\frac{k_{2}}{k_{2}+1} x_{0}^{2}+k_{2}^{2}}>\sqrt{x_{0}^{2}+k_{1}^{2}}$. Then by the Turán-type inequality (3.9) we have $w_{k_{2}}\left(x_{0}\right)>w_{k_{1}}\left(x_{0}\right)$. 
Let $v_{k}(x)=\frac{K_{k}^{\prime}(x)}{K_{k}(x)}$; then $v_{k}(x)$ satisfies

$$
v_{k}^{\prime}=-v_{k}^{2}-\frac{1}{x} v_{k}+1+\frac{k^{2}}{x^{2}} .
$$

Lemma A.2. There exists an $x_{0}, 0<x_{0} \ll 1$, such that $v_{k_{2}}\left(x_{0}\right)<v_{k_{1}}\left(x_{0}\right)$ for all $k_{2}>k_{1} \geq 0$.

Proof. We cannot prove this result when $1>k_{2}>k_{1} \geq 0$ using the technique applied in Lemma A.1 since the Turán-type inequality (3.10) is valid only for $k>1$. We thus use a different approach, which could also be used to prove Lemma A.1. Using 9.6.26 and 9.6.9 in [1] for $k_{2}>k_{1} \geq 0$, we have as $x \rightarrow 0$

$$
\begin{aligned}
\frac{K_{k_{2}}^{\prime}(x)}{K_{k_{2}}(x)}-\frac{K_{k_{1}}^{\prime}(x)}{K_{k_{1}}(x)} & =-\frac{K_{k_{2}+1}(x)}{K_{k_{2}}(x)}+\frac{k_{2}}{x}-\left(-\frac{K_{k_{1}+1}(x)}{K_{k_{1}}(x)}+\frac{k_{1}}{x}\right) \\
& \sim \frac{2}{x}\left(\frac{\Gamma\left(k_{1}+1\right)}{\Gamma\left(k_{1}\right)}-\frac{\Gamma\left(k_{2}+1\right)}{\Gamma\left(k_{2}\right)}\right)+\frac{k_{2}-k_{1}}{x}=-\frac{k_{2}-k_{1}}{x}<0 .
\end{aligned}
$$

Therefore, there exists an $x_{0}>0$ small enough such that $\frac{K_{k_{2}}^{\prime}\left(x_{0}\right)}{K_{k_{2}}\left(x_{0}\right)}<\frac{K_{k_{1}}^{\prime}\left(x_{0}\right)}{K_{k_{1}}\left(x_{0}\right)}$, which we had to prove.

TheOrem A.3. For $x>0, w_{k}(x)$ and $-v_{k}(x)$ are strictly increasing functions of $k$ when $k \geq 0$.

Proof. We only prove the result for $w_{k}(x)$; the proof for $v_{k}(x)$ is similar. For any $k_{2}>k_{1} \geq 0, w_{k_{2}}(x)$ is the solution of $w^{\prime}=f_{k_{2}}(x, w)$ with initial value $\xi_{2}:=w_{k_{2}}\left(x_{0}\right)$, and $w_{k_{1}}(x)$ is the solution of $w^{\prime}=f_{k_{1}}(x, w)$ with initial value $\xi_{1}=w_{k_{1}}\left(x_{0}\right)$, where, by Lemma A.1, $x_{0}$ is chosen such that $\xi_{2}>\xi_{1}$. We denote by $\phi$ the solution of $w^{\prime}=f_{k_{1}}(x, w)$ with initial value $\phi\left(x_{0}\right)=\xi_{2}$. Since $f_{k_{2}}(x, w)>f_{k_{1}}(x, w)$, by a Chaplygin-type comparison theorem [9], we have

$$
w_{k_{2}}(x)>\phi(x), x>x_{0} .
$$

Notice now that $\phi$ and $w_{k_{1}}$ are solutions of $w^{\prime}=f_{k_{1}}(x, w)$ with initial values $\xi_{2}>\xi_{1}$, and hence by the uniqueness of solutions of $w^{\prime}=f_{k_{1}}(x, w)$ (see [1]), we have

$$
\phi(x) \geq w_{k_{1}}(x), x>x_{0} .
$$

By Lemma A.1, $x_{0}$ can be taken small enough, and combining (A.3) and (A.4) the result follows.

Acknowledgments. The second author thanks the University of Geneva for hosting his extended research stay. We thank the anonymous referees for their constructive suggestions and useful comments, which have substantially improved our paper.

\section{REFERENCES}

[1] M. Abramowitz And I. A. Stegun, Handbook of Mathematical Functions with Formulas, Graphs, and Mathematical Tables, Dover, New York, 1972.

[2] Y. Achdou and F. NATAF, An iterated tangential filtering decomposition, Numer. Linear Algebra Appl., 10 (2003), pp. 511-539.

[3] E. Audusse, P. Dreyfuss, and B. Merlet, Optimized Schwarz waveform relaxation for the primitive equations of the ocean, SIAM J. Sci. Comput., 32 (2010), pp. 2908-2936.

[4] A. Baricz and S. Ponnusamy, On Turán type inequalities for modified Bessel functions, Proc. Amer. Math. Soc., 141 (2013), pp. 523-532. 
[5] H. Barucq, M.J. Gander, And Y. Xu, On the influence of curvature on transmission conditions, in Domain Decomposition Methods in Science and Engineering XXI, Lect. Notes Comput. Sci. Eng., Springer, Berlin, 2013, pp. 279-286.

[6] J.-D. Benamou And B. Desprès, A domain decomposition method for the Helmholtz equation and related optimal control problems, J. Comput. Phys., 136 (1997), pp. 68-82.

[7] D. Bennequin, M. J. Gander, and L. Halpern, A homographic best approximation problem with application to optimized Schwarz waveform relaxation, Math. Comp., 78 (2009), pp. 185-223.

[8] M. El Bouajaji, V. Dolean, M. J Gander, and S. Lanteri, Optimized Schwarz methods for the time-harmonic Maxwell equations with damping, SIAM J. Sci. Comput., 34 (2012), pp. A2048-A2071.

[9] S. A. Chaplygin, New Methods in the Approximate Integration of Differential Equations, Gostiekhizdat, Moscowa, 1950 (in Russian).

[10] Z. Chen, M. J. Gander, And H. Zhang, On the relation between optimized Schwarz methods and source transfer, Domain Decomposition Methods in Science and Engineering XXII, 2014, accepted.

[11] Z. Chen And X. XIAng, A source transfer domain decomposition method for Helmholtz equations in unbounded domain, SIAM J. Numer. Anal., 51 (2013), pp. 2331-2356.

[12] B. Després, Décomposition de domaine et probleme de Helmholtz, C. R. Acad. Sci. Paris Ser. 1 Math., 311 (1990), pp. 313-316.

[13] V. Dolean, M. J. Gander, and L Gerardo-Giorda, Optimized Schwarz methods for Maxwell's equations, SIAM J. Sci. Comput., 31 (2009), pp. 2193-2213.

[14] B. Engquist and L. Ying, Sweeping preconditioner for the Helmholtz equation: Moving perfectly matched layers, Multiscale Model. Simul., 9 (2011), pp. 686-710.

[15] B. Engquist and H.-K. ZhaO, Absorbing boundary conditions for domain decomposition, Appl. Numer. Math., 27 (1998), pp. 341-365.

[16] M. J. Gander, AILU: A preconditioner based on the analytic factorization of the elliptic operator, Numer. Linear Algebra Appl., 7 (2000), pp. 505-526.

[17] M. J. Gander, Optimized Schwarz methods, SIAM J. Numer. Anal., 44 (2006), pp. 699-731.

[18] M. J. Gander, Schwarz methods over the course of time, Electron. Trans. Numer. Anal., 31 (2008), pp. 228-255.

[19] M. J. Gander and L. Halpern, Absorbing boundary conditions for the wave equation and parallel computing, Math. Comp., 74 (2005), pp. 153-176.

[20] M. J. Gander and L. Halpern, Optimized Schwarz waveform relaxation methods for advection reaction diffusion problems, SIAM J. Numer. Anal., 45 (2007), pp. 666-697.

[21] M. J. Gander, L. Halpern, And F. NAtaf, Optimal Schwarz waveform relaxation for the one dimensional wave equation, SIAM J. Numer. Anal., 41 (2003), pp. 1643-1681.

[22] M. J. Gander, F. Magoulès, And F. NATAF, Optimized Schwarz methods without overlap for the Helmholtz equation, SIAM J. Sci. Comput., 24 (2002), pp. 38-60.

[23] M. J. Gander and F. NATAF, An incomplete LU preconditioner for problems in acoustics, J. Comput. Acoust., 13 (2005), pp. 455-476.

[24] M. J. Gander and Y. Xu, Optimized Schwarz Methods with Nonoverlapping Circular Domain Decomposition, in preparation.

[25] M. J. Gander And Y. XU, Optimized Schwarz algorithm with two-sided transmission conditions in an unsymmetric domain decomposition, submitted to Domain Decomposition Methods in Science and Engineering XXII, (2014).

[26] G. Gigante, M. Pozzoli, and C. Vergara, Optimized Schwarz methods for the diffusionreaction problem with cylindrical interfaces, SIAM J. Numer. Anal., 51 (2013), pp. 34023430.

[27] J.-H. Kimn, A convergence theory for an overlapping Schwarz algorithm using discontinuous iterates, Numer. Math., 100 (2005), pp. 117-139.

[28] P.-L. Lions, On the Schwarz alternating method. III: A variant for nonoverlapping subdomains, in Proceedings of the 3rd International Symposium on Domain Decomposition Methods for Partial Differential Equations, Vol. 6, SIAM, Philadelphia, 1990, pp. 202-223.

[29] S. LoISEL, Condition number estimates for the nonoverlapping optimized Schwarz method and the 2-Lagrange multiplier method for general domains and cross points, SIAM J. Numer. Anal., 51 (2013), pp. 3062-3083.

[30] S. Loisel And D. B. Szyld, On the geometric convergence of optimized Schwarz methods with applications to elliptic problems, Numer. Math., 114 (2010), pp. 697-728.

[31] S. H. LuI, A Lions non-overlapping domain decomposition method for domains with an arbitrary interface, IMA J. Numer. Anal., 29 (2009), pp. 332-349. 
[32] S. H. LuI, Convergence estimates for an higher order optimized Schwarz method for domains with an arbitrary interface, J. Comput. Appl. Math., 235 (2010), pp. 301-314.

[33] F. Magoulès, P. Iványi, And B.H.V. Topping, Non-overlapping Schwarz methods with optimized transmission conditions for the Helmholtz equation, Comput. Methods Appl. Mech. Engrg., 193 (2004), pp. 4797-4818.

[34] V. MARTin, An optimized Schwarz waveform relaxation method for the unsteady convection diffusion equation in two dimensions, Appl. Numer. Math., 52 (2005), pp. 401-428.

[35] F. NATAF, Optimized Schwarz methods, in Domain Decomposition Methods in Science and Engineering XVIII, Springer, New York, 2009, pp. 233-240.

[36] F. Nobile, M. Pozzoli, and C. Vergara, Time accurate partitioned algorithms for the solution of fluid-structure interaction problems in haemodynamics, Comput. Fluids, 86 (2013), pp. 470-482.

[37] Z. Peng And J.-F. LeE, Non-conformal domain decomposition method with second-order transmission conditions for time-harmonic electromagnetics, J. Comput. Phys., 229 (2010), pp. 5615-5629.

[38] Z. Peng, V. Rawat, And J.-F. Lee, One way domain decomposition method with second order transmission conditions for solving electromagnetic wave problems, J. Comput. Phys., 229 (2010), pp. 1181-1197.

[39] A. Qaddouri, L. Laayouni, S. Loisel, J. Côté, and M. J. Gander, Optimized Schwarz methods with an overset grid for the shallow-water equations: Preliminary results, Appl. Numer. Math., 58 (2008), pp. 459-471.

[40] L. QIN AND X. XU, Optimized Schwarz methods with Robin transmission conditions for parabolic problems, SIAM J. Sci. Comput., 31 (2008), pp. 608-623.

[41] A. A. Rodríguez and L. Gerardo-Giorda, New nonoverlapping domain decomposition methods for the harmonic Maxwell system, SIAM J. Sci. Comput., 28 (2006), pp. 102-122.

[42] C. C. Stolk, A rapidly converging domain decomposition method for the Helmholtz equation, J. Comput. Phys., 241 (2013), pp. 240-252. 